\title{
A Semilinear Fourth Order Elliptic Problem with Exponential Nonlinearity
}

\author{
Gianni Arioli* - Filippo Gazzola* \\ Hans-Christoph Grunau - Enzo Mitidieri
}

16th October 2003

\begin{abstract}
We study a semilinear fourth order elliptic problem with exponential nonlinearity. Motivated by a question raised in [Li], we partially extend known results for the corresponding second order problem. Several new difficulties arise and many problems still remain to be solved. We list the ones we feel particularly interesting in the final section. Mathematics Subject Classification: 35J65; 35J40.
\end{abstract}

\section{Introduction}

In the last forty years a great deal has been written about existence and multiplicity of solutions to nonlinear second order elliptic problems in bounded and unbounded domains of $\mathbb{R}^{n}(n \geq 2)$. Important achievements on this topic have been obtained by applying various combinations of analytical techniques, among all of them we only mention the variational and topological methods. For the latter, especially when the main interest is focused on existence of positive solutions, the fundamental tool which has been used is the maximum principle [A1] and its consequences [GNN].

For higher order problems, a possible failure of the maximum principle causes several technical difficulties. This fact is very likely the reason why the knowledge on higher order nonlinear problems is far from being reasonably complete as in the second order case.

One of the most interesting and intensively studied second order model problems that exhibits several peculiar features of most nonlinear elliptic equations is the so-called Gelfand problem [G, Section 15],

$$
\left\{\begin{array}{lr}
-\Delta u=\lambda e^{u} & \text { in } \Omega \\
u=0 & \text { on } \partial \Omega .
\end{array}\right.
$$

Here $\Omega$ is a bounded smooth domain in $\mathbb{R}^{n}(n \geq 3)$ and $\lambda \geq 0$ is a parameter. This problem appears in connection with combustion theory $[\mathrm{G}, \mathrm{JL}]$ and stellar structure $[\mathrm{C}]$. From a

*The work of G.A. and F.G. was supported by the MURST project "Metodi Variazionali ed Equazioni Differenziali non Lineari." 
mathematical point of view, one of the main interests is that it may have both unbounded (singular) solutions and bounded (regular) solutions, see [BV, GMP, MP2]: by the results in $[\mathrm{CR}, \mathrm{BCMR}]$ it is known that there exists $\lambda^{*}>0$ such that if $\lambda>\lambda^{*}$ there exists no solution of (1) (neither regular nor singular) while if $0 \leq \lambda<\lambda^{*}$ there exists a minimal regular solution $U_{\lambda}$ of (1) and the map $\lambda \mapsto U_{\lambda}$ is smooth and increasing. In the unit ball $B$, the bifurcation picture of radial solutions is rather complete. There is always a singular solution $u_{\sigma}:=-2 \log |x|$ with corresponding parameter $\lambda_{\sigma}=2(n-2)$. If $n \geq 10$ the solution branch consists only of minimal solutions and terminates at $\lambda^{*}=\lambda_{\sigma}$ in the singular solution. If $3 \leq n \leq 9$, then $\lambda^{*}>\lambda_{\sigma}$ and the extremal point $\left(\lambda^{*}, U_{*}\right)$ is a turning point. The branch bends back and meanders infinitely many times around $\lambda_{\sigma}$, while approaching the singular solution $u_{\sigma}$. We refer to [BV, Figure 1] for the pictures. The interested reader may see also $[\mathrm{BE}]$ for an account on motivations and related results.

Some interesting generalizations of (1) have been considered in the framework of second order quasilinear operators. We refer to $[\mathrm{GPP}]$ for equations associated to the $p$-Laplace operator and to $[\mathrm{J}, \mathrm{JS}]$ for the case of the $k$-Hessian operator.

The aim of this paper is to give a contribution to the solution of a special case of a problem formulated in [Li, Section $4.2(\mathrm{c})$ ], namely:

Is it possible to obtain a description of the solution set for higher order semilinear equations associated to exponential nonlinearities?

Recently, the interest on higher order nonlinear problems due to its exciting and promising developments became increasingly evident especially for fourth order equations [PT]. Following this trend, in this paper we shall consider the fourth order version of (1), a semilinear elliptic problem which involves the biharmonic operator, more precisely

$$
\left\{\begin{array}{l}
\Delta^{2} u=\lambda e^{u} \quad \text { in } B \\
u=\frac{\partial u}{\partial \mathbf{n}}=0 \quad \text { on } \partial B
\end{array}\right.
$$

Here $B$ denotes the unit ball in $\mathbb{R}^{n}(n \geq 5)$ centered at the origin and $\frac{\partial}{\partial \mathbf{n}}$ the differentiation with respect to the exterior unit normal i.e. in radial direction; $\lambda \geq 0$ is a parameter. We are interested in two kinds of solutions of $\left(P_{\lambda}\right)$, regular solutions and singular solutions, see Definition 1 in the next section. We restrict our attention to the case $n \geq 5$ where the nonlinearity is supercritical. In low dimensions $1 \leq n \leq 4$ the problem is subcritical and has a different behaviour, see Remark 4 at the end of the following section.

Many techniques, familiar from second order equations like the maximum principle, are not available here. But since we restrict ourselves to the ball, at least a comparison principle is available, see Lemma 1 below. Moreover, in fourth order equations, one usually does not succeed in finding suitable nontrivial auxiliary functions satisfying again a differential inequality. This is a serious difficulty in proving Theorem 1 (cf. the proof of [BCMR, Theorem $3]$ ) and it is overcome by carefully exploiting the properties of the exponential nonlinearity and the construction of minimal solutions, basing upon the already mentioned comparison principle. Finally, when looking for radial solutions, one may perform a phase space analysis for the corresponding system of ODEs. Here, the phase space is no longer two dimensional, where the topology is relatively simple and the Poincaré-Bendixson-theory is available, but we have to work in a four-dimensional phase space. Some of the resulting difficulties could be overcome only with computer assistance. 
This paper is organized as follows: in the next section we state some definitions and the main results contained in this work (see Theorems 1-5 below). The content of Section 3 through Section 7 is devoted to the proofs of these theorems. Section 8 contains some results on the stability of regular solutions of $\left(P_{\lambda}\right)$ and a list of open problems that we consider of some interest and related to the main results of this paper. Finally in Section 9 we describe the algorithm used in the computer assisted proof of Theorem 4.

\section{Main results}

We first make precise in which sense we intend a function to solve $\left(P_{\lambda}\right)$. For this purpose, we fix some exponent $p$ with $p>\frac{n}{4}$ and $p \geq 2$. The definitions and results below do not depend on the special choice of $p$.

Definition 1. We say that $u \in L^{2}(B)$ is a solution of $\left(P_{\lambda}\right)$ if $e^{u} \in L^{1}(B)$ and

$$
\int_{B} u \Delta^{2} v=\lambda \int_{B} e^{u} v \quad \text { for all } v \in W^{4, p} \cap H_{0}^{2}(B) .
$$

We say that a solution $u$ of $\left(P_{\lambda}\right)$ is regular (resp. singular) if $u \in L^{\infty}(B)$ (resp. $u \notin L^{\infty}(B)$ ).

Clearly, according to this definition, regular and singular solutions exhaust all possible solutions. Note that by standard regularity theory for the biharmonic operator (see [ADN]), any regular solution $u$ of $\left(P_{\lambda}\right)$ satisfies $u \in C^{\infty}(\bar{B})$. Note also that by the positivity preserving property of $\Delta^{2}$ in the ball [B] any solution of $\left(P_{\lambda}\right)$ is positive, see also Lemmas 1 and 3 below for a generalized statement. This property is known to fail in general domains. For this reason, we restrict ourselves to balls also in Theorems 1 and 2; cf. also Open Problem 8 in Section 8.

We also need the notion of minimal solution:

Definition 2. We call a solution $U_{\lambda}$ of $\left(P_{\lambda}\right)$ minimal, if $U_{\lambda} \leq u_{\lambda}$ a.e. in $B$ for any further solution $u_{\lambda}$ of $\left(P_{\lambda}\right)$.

In order to state our results, we denote by $\lambda_{1}>0$ the first eigenvalue for the biharmonic operator with Dirichlet boundary conditions

$$
\begin{cases}\Delta^{2} u=\lambda_{1} u & \text { in } B \\ u=\frac{\partial u}{\partial \mathbf{n}}=0 & \text { on } \partial B\end{cases}
$$

it is known from the mentioned positivity preserving property and Jentzsch's (or KreinRutman's) theorem that $\lambda_{1}$ is isolated and simple and that the corresponding eigenfunctions do not change sign.

We may now state

Theorem 1. There exists

$$
\lambda^{*} \in\left[14.72(n-1)(n-3), \frac{\lambda_{1}}{e}\right)
$$


such that

(i) $\left(P_{\lambda}\right)$ admits a minimal regular solution $U_{\lambda}$ for all $\lambda\left\langle\lambda^{*}\right.$ and no solutions if $\lambda>\lambda^{*}$.

(ii) The map $\lambda \mapsto U_{\lambda}(x)$ is strictly increasing for all $x \in B$. Moreover, there exists a solution $U_{*}$ of $\left(P_{\lambda^{*}}\right)$ which is the pointwise limit of $U_{\lambda}$ as $\lambda \uparrow \lambda^{*}$.

(iii) $U_{\lambda} \rightarrow U_{*}$ in the norm topology of $H_{0}^{2}(B)$ as $\lambda \uparrow \lambda^{*}$.

(iv) The extremal solution $U_{*}$ and all the minimal solutions $U_{\lambda}\left(\right.$ for $\left.\lambda<\lambda^{*}\right)$ are radially symmetric and radially decreasing.

It is remarkable that at $\lambda^{*}$ there is an immediate switch from existence of regular minimal solutions to nonexistence of any (even singular) solution. The only possibly singular minimal solution corresponds to $\lambda=\lambda^{*}$. This result is known from [BCMR] for the second order problem (1), but the method used there may not be carried over to fourth order problems. Nevertheless, the result extends to the biharmonic case. The proof is given in Lemma 5 below.

We may also characterize the uniform convergence to 0 of $U_{\lambda}$ as $\lambda \rightarrow 0$ by giving the precise rate of its extinction:

Theorem 2. For all $\lambda \in\left(0, \lambda^{*}\right)$ let $U_{\lambda}$ be the minimal solution of $\left(P_{\lambda}\right)$ and let

$$
V_{\lambda}(x)=\frac{\lambda}{8 n(n+2)}\left[1-|x|^{2}\right]^{2} .
$$

Then, $U_{\lambda}(x)>V_{\lambda}(x)$ for all $\lambda<\lambda^{*}$ and all $|x|<1$, and

$$
\lim _{\lambda \rightarrow 0} \frac{U_{\lambda}(x)}{V_{\lambda}(x)}=1 \quad \text { uniformly w.r.t. } x \in B .
$$

A complete result in the spirit of Gidas-Ni-Nirenberg [GNN] does not hold for fourth order equations under Dirichlet boundary conditions. It has been recently proved by Sweers in $[\mathrm{Sw}]$ that for general semilinear autonomous biharmonic equations in a ball under Dirichlet boundary conditions we may have positive radially symmetric solutions which are not radially decreasing, provided the right hand side is not positive everywhere. This phenomenon may not occur in our situation, however, it is not known whether any smooth solution of $\left(P_{\lambda}\right)$ is radially symmetric. Moreover, also in the second order case it is not known whether singular solutions are always radially symmetric. Nevertheless, Theorem 1 suggests to pay particular attention to radially symmetric solutions. In this context, we put $r=|x|$ and consider the functions $u=u(r)$.

First of all, we introduce a new notion of solution which seems to be the natural framework for radially symmetric solutions:

Definition 3. We say that a radial singular solution $u=u(r)$ of $\left(P_{\lambda}\right)$ is weakly singular if the limit $\lim _{r \rightarrow 0} r u^{\prime}(r)$ exists.

We do not know whether every singular solution is also weakly singular. In the second order case, Joseph-Lundgren [JL] reduce (1) to a system of two ODE's and study its phase portrait in $\mathbb{R}^{2}$; using Bendixson's Theorem, they show that singular solutions are also weakly singular. For the fourth order equation $\left(P_{\lambda}\right)$ a similar argument should be carried out in $\mathbb{R}^{4}$ (see Section 3) where a general result of Bendixson's type does not hold. Therefore, the 
equivalence between singular and weakly singular solutions seems out of reach in our context, see Open Problem 5 in Section 8.

If we seek radially symmetric solutions, we may rewrite problem $\left(P_{\lambda}\right)$ as $(0<r \leq 1)$

$$
\left\{\begin{array}{l}
\frac{d^{4} u}{d r^{4}}+\frac{2(n-1)}{r} \frac{d^{3} u}{d r^{3}}+\frac{(n-1)(n-3)}{r^{2}} \frac{d^{2} u}{d r^{2}}-\frac{(n-1)(n-3)}{r^{3}} \frac{d u}{d r}=\lambda e^{u(r)} \\
u(1)=0 \\
\left.\frac{d u}{d r}\right|_{r=1}=0
\end{array}\right.
$$

In [GPP, JL, MP2] the second order equation (1) was reduced to a system of two autonomous ordinary differential equations. Here, we reduce (4) to a system of four equations. First, we make the change of variables

$$
s=\log r \quad v(s)=u\left(e^{s}\right) \quad s \in(-\infty, 0]
$$

so that (4) becomes

$$
\left\{\begin{array}{l}
\frac{d^{4} v}{d s^{4}}+2(n-4) \frac{d^{3} v}{d s^{3}}+\left(n^{2}-10 n+20\right) \frac{d^{2} v}{d s^{2}}-2(n-2)(n-4) \frac{d v}{d s}=\lambda e^{4 s+v(s)} \\
v(0)=0 \\
\left.\frac{d v}{d s}\right|_{s=0}=0
\end{array}\right.
$$

then, we set

$$
\left\{\begin{array}{l}
v_{1}(s)=v^{\prime}(s)+4 \\
v_{2}(s)=-v^{\prime \prime}(s)-(n-2) v^{\prime}(s) \\
v_{3}(s)=-v^{\prime \prime \prime}(s)+(4-n) v^{\prime \prime}(s)+2(n-2) v^{\prime}(s) \\
v_{4}(s)=-\lambda e^{v(s)+4 s}
\end{array}\right.
$$

Finally, we obtain the following (nonlinear) differential system:

$$
\left\{\begin{array}{l}
v_{1}^{\prime}(s)=(2-n) v_{1}(s)-v_{2}(s)+4(n-2) \\
v_{2}^{\prime}(s)=2 v_{2}(s)+v_{3}(s) \\
v_{3}^{\prime}(s)=(4-n) v_{3}(s)+v_{4}(s) \\
v_{4}^{\prime}(s)=v_{1}(s) v_{4}(s)
\end{array}\right.
$$

with initial conditions

$$
v_{1}(0)=4, \quad v_{4}(0)=-\lambda .
$$

It turns out that (8) admits only the two stationary points $P_{1}=(4,0,0,0)$ and $P_{2}=$ $(0,4 n-8,16-8 n,-8(n-2)(n-4))$, see Section 3.1. Then, in Section 3.2, we prove the following result:

Theorem 3. Let $u=u(r)$ be a radial solution of $\left(P_{\lambda}\right)$ and let

$$
V(s)=\left(v_{1}(s), v_{2}(s), v_{3}(s), v_{4}(s)\right)
$$


be the corresponding trajectory relative to (8). Then:

(i) $u$ is regular (i.e. $u \in L^{\infty}(B)$ ) if and only if

$$
\lim _{s \rightarrow-\infty} V(s)=P_{1}
$$

(ii) $u$ is weakly singular if and only if

$$
\lim _{s \rightarrow-\infty} V(s)=P_{2} .
$$

Our next results concern the existence of weakly singular solutions and a lower bound $\lambda_{\min }^{*}$ on the value of $\lambda^{*}$. For all $n=5, \ldots, 16$ we prove the existence of $\lambda_{\sigma}$ such that $\left(P_{\lambda_{\sigma}}\right)$ admits a weakly singular solution, we provide a lower and upper bound on the value of $\lambda_{\sigma}$. For all $n=5, \ldots, 16$ let $\lambda_{\sigma}^{\min }$ and $\lambda_{\sigma}^{\max }$ be given in Table (10) and for all $n=5, \ldots, 10$ let $\lambda_{\min }^{*}$ be given in Table (10).

Theorem 4. For all $n=5, \ldots, 16$ there exists $\lambda_{\sigma} \in\left[\lambda_{\sigma}^{\min }, \lambda_{\sigma}^{\max }\right]$ such that $\left(P_{\lambda_{\sigma}}\right)$ admits a weakly singular solution $U_{\sigma}$. In particular, $\lambda_{\sigma}>8(n-2)(n-4)$.

For all $n=5, \ldots, 10$ the value of $\lambda^{*}$ is larger than $\lambda_{\min }^{*}$.

In Section 6 we use Theorem 3 to show that Theorem 4 is equivalent to some intersection properties of the unstable manifolds of $P_{1}$ and $P_{2}$ with the hyperplane $v_{1}=4$. The remaining part of the proof of Theorem 4 is divided in two parts. First, in Section 6 a rigorous bound on the location of the unstable manifold close to the stationary point is obtained by analytical methods. Then, the intersection of the manifold with the hyperplane and its location are proved by a computer assisted algorithm, see Section 9. Let us explain exactly what we mean by a computer assisted proof:

Definition 4. A proof is called computer assisted if it consists in finitely many elementary operations, but their number is so large that, although each step may be written down explicitly, it is only practical to perform such operations with a computer.

We believe that a weakly singular solution exists in any dimension $n \geq 5$, but since our type of proof requires a finite number of steps for each value of $n$, we cannot prove this conjecture. We performed the computer assisted proof for $n=5, \ldots, 16$ because the "interesting" phenomena of $\left(P_{\lambda}\right)$ arise in these dimensions.

We expect the "singular parameter" $\lambda_{\sigma}$ and the singular solution to be unique. However, also for this statement, we do not yet have a proof. See Open Problem 3 in Section 8 below.

The following table summarizes our results: $\lambda^{*}$ and $\lambda_{\sigma}$ are the best, purely numerical, estimates for the values, up to two decimal digits, while the numbers $\lambda_{\sigma}^{\min }, \lambda_{\sigma}^{\max }$ and $\lambda_{\min }^{*}$ are rigorously computed values as stated in Theorem 4. 


$\begin{array}{cccccc}n & \lambda_{\sigma} & \lambda^{*} & \lambda_{\sigma}^{\min } & \lambda_{\sigma}^{\max } & \lambda_{\min }^{*} \\ 5 & 113.19 & 236.49 & 113.11 & 113.26 & 235.89 \\ 6 & 260.82 & 362.10 & 260.72 & 260.86 & 361.34 \\ 7 & 449.55 & 524.70 & 449.45 & 449.60 & 523.16 \\ 8 & 679.45 & 728.36 & 679.04 & 679.55 & 724.50 \\ 9 & 950.28 & 976.66 & 949.58 & 950.49 & 969.81 \\ 10 & 1261.79 & 1272.09 & 1260.71 & 1262.23 & 1268.48 \\ 11 & 1613.78 & 1615.77 & 1610.89 & 1615.30 & \\ 12 & 2006.09 & 2006.11 & 1997.53 & 2010.41 & \\ 13 & 2438.60 & 2438.60 & 2403.42 & 2457.15 & \\ 14 & 2911.21 & 2911.21 & 2843.32 & 2947.17 & \\ 15 & 3423.83 & 3423.83 & 3260.54 & 3514.51 & \\ 16 & 3976.40 & 3976.40 & 3597.37 & 4211.88 & \end{array}$

Remark 1. We point out that both the approximate numerical computation and the computation with rigorous estimate on the error for $\lambda^{*}$ become very difficult as $n$ increases. For this reason the best rigorous estimate we have on $\lambda_{\min }^{*}$ for $n \geq 11$ is nothing but for $\lambda_{\sigma}^{\min }$, while the best numerical estimate we have on $\lambda^{*}$ for $n \geq 13$ is $\lambda_{\sigma}$. These values of $n$ may be improved with a more accurate algorithm, but we do not feel that this would lead to a qualitative improvement of the result.

From Table (10) we immediately get

Corollary 1. For all $n=5, \ldots, 10$ we have $\lambda_{\sigma}<\lambda^{*}$.

Remark 2. We have numerical evidence that $\lambda_{\sigma}<\lambda^{*}$ for $n=11,12$ as well, but $\lambda^{*}-\lambda_{\sigma}$ is much smaller than the rigorous estimate of the numerical error, therefore we do not have a proof. For $n \geq 13$ the values of $\lambda_{\sigma}$ and $\lambda^{*}$ are closer than the numerical error, therefore we cannot even provide a conjecture supported by numerical evidence. If one could show uniqueness of the singular parameter $\lambda_{\sigma}$ and that in fact $\lambda_{\sigma}<\lambda^{*}$ in dimensions $n \leq 12$, one could conclude that here, the extremal solution $U_{*}$ is either regular or "strongly singular" (i.e. $\lim _{r \rightarrow 0} r u^{\prime}(r)$ does not exist). For $n \geq 13$ we expect the extremal solution $U_{*}$ to be weakly singular. See Open Problems 3, 4 and 5 in Section 8.

To complete the numerical inspection of the problem we provide the following picture, which shows the (regular) solution for $n=5$ and $\lambda=\lambda^{*}$ : 


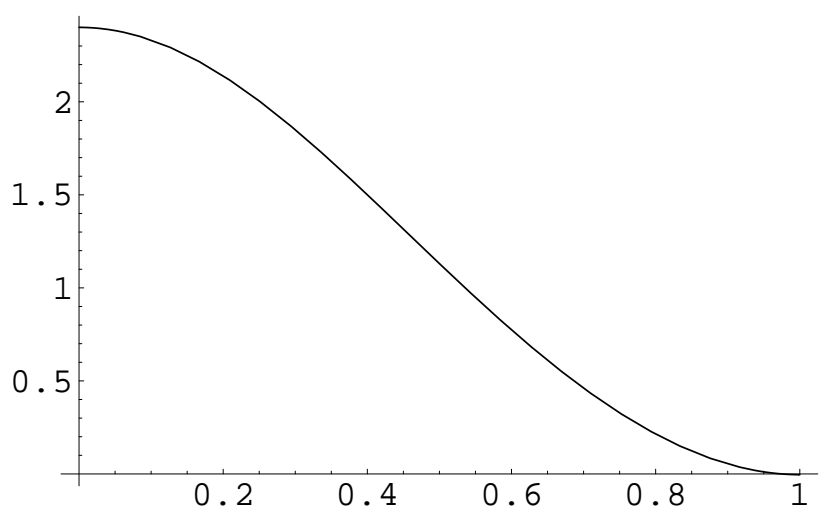

Theorem 3 states that any weakly singular solution $u=u(r)$ of (4) corresponds to a (weakly singular) solution $v=v(s)$ of $(6)$ which satisfies $v(s) \approx-4 s$ as $s \rightarrow-\infty$ : this is because $\bar{v}(s)=-4 s$ is precisely the stationary point $P_{2}$. Hence, as a further consequence of Theorem 3 , we have that any weakly singular solution $U_{\sigma}$ behaves asymptotically like $-4 \log r$ as $r \rightarrow 0$. Moreover, as may be checked by a simple calculation, the function $r \mapsto-4 \log r$ solves the equation and the first boundary condition in $\left(P_{\lambda}\right)$ for $\lambda=8(n-2)(n-4)$ but not the second boundary condition (recall also $\lambda_{\sigma}>8(n-2)(n-4)$ by Theorem 4$)$. Contrary to what happens for the second order equation (1), the explicit form of the radial weakly singular solution seems not simple to be determined, see also Proposition 1 below. To this end, we characterize it further by means of the following

Theorem 5. Let $U_{\sigma}$ be a weakly singular solution with $\lambda_{\sigma}>8(n-2)(n-4)$ as it is obtained in Theorem 4 for $5 \leq n \leq 16$. Then,

$$
U_{\sigma}(r)=-4 \log r+W(r)
$$

where $W$ is a bounded function satisfying

$$
\lim _{r \rightarrow 0} W(r)=W_{0}:=\log \frac{8(n-2)(n-4)}{\lambda_{\sigma}}<0
$$

and (at least) one of the two following facts holds true:

(i) $W(r)-W_{0}$ changes sign infinitely many times in any neighbourhood of $r=0$;

(ii) $W(r) \geq \max \left[W_{0}, 2 r^{2}-2\right]$ for all $r \in(0,1]$.

If $n \geq 13$, case (ii) necessarily occurs.

Finally, the function $W(r)$ is not analytic, i.e. not a convergent power series in $r^{2}$ close to $r_{0}=0$.

Remark 3. It is quite surprising that the asymptotic behaviour of weakly singular solutions of $\left(P_{\lambda}\right)$ is the same as the one of the quasilinear equation $-\Delta_{4} u=\lambda e^{u}$, see [GPP]. Here $-\Delta_{p}$ denotes the $p$-Laplace operator.

We conclude this section with a short remark concerning the behaviour of $\left(P_{\lambda}\right)$ in low dimensions.

Remark 4. In dimensions $1 \leq n \leq 4$ the problem is subcritical and has a different behaviour. In particular, there are no singular solutions. 
The minimal solution is constructed as in the present paper. There is a parameter $\lambda^{*}>0$ such that for any $\lambda \in\left(0, \lambda^{*}\right)$ there is precisely one minimal stable positive solution. Taking this one as "trivial" solution, with help of variational techniques (which only apply in a subcritical setting) one finds a second positive "large" solution, above the minimal solution and unstable. For $\lambda>\lambda^{*}$ there is no positive solution. Concerning the bifurcation diagram, one expects a smooth branch emanating from 0 , extending until $\lambda^{*}$, where it bends back and approaches $\lambda=0$, while the $L^{\infty}$ norm of the solutions blows up. See also [We, Wi].

\section{Characterization of regular and weakly singular ra- dial solutions}

In this section we perform a phase space analysis for the system (8), which corresponds to the radial version of $\left(P_{\lambda}\right)$. This gives some insight on which behaviour of regular and of weakly singular radial solutions may be expected in dependence on the space dimension. These results are essential for the proofs of Theorems 4 and 5. For the proofs of Theorems 1 and 2 one may directly skip to Sections 4 and 5 .

\subsection{Analysis of the stationary points}

It is easy to verify that system (8) has only two stationary points:

$$
P_{1}=(4,0,0,0) \quad \text { and } \quad P_{2}=(0,4 n-8,16-8 n,-8(n-2)(n-4)) .
$$

In order to linearize $(8)$ in a neighbourhood of $P_{1}$, we must just replace $(8)_{4}$ with

$$
v_{4}^{\prime}(s)=4 v_{4}(s)
$$

Then, the linearized system has two distinct positive eigenvalues $\mu_{1}=2, \mu_{2}=4$ and two distinct negative ones $\mu_{3}=2-n, \mu_{4}=4-n$. We conclude that $P_{1}$ is a hyperbolic point independently of the dimension.

Eigenvectors corresponding to the positive eigenvalues $\mu_{1}, \mu_{2}$ in the neighbourhood of $P_{1}$ have the form

$$
\alpha_{1}(1,-n, 0,0) \quad \text { and } \quad \alpha_{2}\left(-1, n+2,2 n+4,2 n^{2}+4 n\right),
$$

where $\alpha_{1}, \alpha_{2} \in \mathbb{R} \backslash\{0\}$. Therefore, the tangent hyperplane to the unstable manifold of $P_{1}$ consists of those points in $\mathbb{R}^{4}$ whose coordinates can be represented as

$$
\left(\alpha_{1}-\alpha_{2},-n \alpha_{1}+(n+2) \alpha_{2},(2 n+4) \alpha_{2},\left(2 n^{2}+4 n\right) \alpha_{2}\right)
$$

with $\alpha_{1}, \alpha_{2} \in \mathbb{R}$.

Similarly, the tangent hyperplane to the stable manifold of $P_{1}$ is spanned by eigenvectors corresponding to negative eigenvalues of the linearized system, that is,

$$
\alpha_{3}(1,0,0,0) \text { and } \alpha_{4}(1,-2,2 n-4,0) \text {, }
$$

where $\alpha_{3}, \alpha_{4} \in \mathbb{R} \backslash\{0\}$. 
Now consider the second critical point $P_{2}$ of (8). In its neighbourhood the linear approximation of $(8)_{4}$ (the only nonlinear equation) takes the form

$$
v_{4}^{\prime}(s)=-8(n-2)(n-4) v_{1}(s) .
$$

Therefore, the eigenvalues of the linearized system in the neighbourhood of $P_{2}$ are the solutions of the fourth order algebraic equation

$$
\nu(\nu-2)(\nu+n-2)(\nu+n-4)-8(n-2)(n-4)=0
$$

hence,

$$
\nu_{1,2,3,4}=\frac{1}{2}\left(4-n \pm \sqrt{M_{1}(n) \pm M_{2}(n)}\right)
$$

where $M_{1}(n)=n^{2}-4 n+8=(n-2)^{2}+4>(n-2)^{2}$ and $M_{2}(n)=4 \sqrt{68-52 n+9 n^{2}}$. Therefore,

$$
\nu_{1}=\frac{1}{2}\left(4-n+\sqrt{M_{1}(n)+M_{2}(n)}\right) \quad \text { and } \quad \nu_{2}=\frac{1}{2}\left(4-n-\sqrt{M_{1}(n)+M_{2}(n)}\right)
$$

are real numbers. It is easy to see that

$$
\nu_{2}<0<\nu_{1} \quad \text { for all } n \geq 4 \text {. }
$$

Moreover, for $5 \leq n \leq 12$, we have $M_{1}(n)-M_{2}(n)<0$, while for $n \geq 13$ there holds $M_{1}(n)-M_{2}(n)>0$. Therefore, for $5 \leq n \leq 12$ the eigenvalues

$$
\nu_{3}=\frac{1}{2}\left(4-n+\sqrt{M_{1}(n)-M_{2}(n)}\right) \quad \text { and } \quad \nu_{4}=\frac{1}{2}\left(4-n-\sqrt{M_{1}(n)-M_{2}(n)}\right)
$$

are complex conjugate with the real part

$$
\operatorname{Re} \nu_{3}=\operatorname{Re} \nu_{4}=\frac{1}{2}(4-n)<0
$$

while for $n \geq 13$ both $\nu_{3}$ and $\nu_{4}$ are real, $\nu_{3}<0$ and $\nu_{4}<0$.

This analysis implies that for all $n \geq 5$ the critical point $P_{2}$ of system (8) is also hyperbolic, but its stable manifold is 3-dimensional and the unstable manifold is 1-dimensional. Moreover, taking into account that for $5 \leq n \leq 12$ we have $\operatorname{Im} \nu_{3}=-\operatorname{Im} \nu_{4} \neq 0$, we deduce from the general theory of critical points (see for example [A2]) that for these values of $n$ (and only for them) trajectories in the stable manifold of $P_{2}$ locally have the form of a spiral.

\subsection{Proof of Theorem 3}

We first consider regular solutions. It will prove to be useful to have the following meaning of $v_{1}, \ldots, v_{4}$ in terms of derivatives of $u$ in mind:

$$
\left\{\begin{array}{l}
v_{1}(s)=e^{s} u^{\prime}\left(e^{s}\right)+4 \\
v_{2}(s)=-e^{2 s} \cdot \Delta u\left(e^{s}\right) \\
v_{3}(s)=-e^{3 s}(\Delta u)^{\prime}\left(e^{s}\right) \\
v_{4}(s)=-\lambda e^{4 s} e^{u\left(e^{s}\right)}
\end{array}\right.
$$


If $u$ is a regular solution of $\left(P_{\lambda}\right)$, then $u, u^{\prime}, \Delta u$ and $(\Delta u)^{\prime}$ stay in particular bounded for $r \searrow 0$, i.e. for $s \rightarrow-\infty$. So, we get immediately from (12) the first part of the statement.

To prove the converse, assume that

$$
\lim _{s \rightarrow-\infty}\left(v_{1}(s), v_{2}(s), v_{3}(s), v_{4}(s)\right)=P_{1}
$$

so that

$$
\lim _{r \searrow 0} r u^{\prime}(r)=\lim _{r \searrow 0} r^{2} \Delta u(r)=\lim _{r \searrow 0} r^{3}(\Delta u)^{\prime}(r)=\lim _{r \searrow 0} r^{4} e^{u(r)}=0 .
$$

The first limit yields in particular that for $r>0$ small enough:

$$
u(r) \leq-\frac{1}{2} \log (r), \quad e^{u(r)} \leq r^{-1 / 2}
$$

Using the differential equation $\left(P_{\lambda}\right)$ and the growth conditions (13) (observe $n>4$ ), we obtain successively for $r$ close to 0 :

$$
(\Delta u)^{\prime}(r)=O\left(r^{1 / 2}\right), \Delta u(r)=O(1), u^{\prime}(r)=O(r), u(r)=O(1) .
$$

That means that $u$ is regular.

Next, we characterize weakly singular solutions. All the limits are intended as $s \rightarrow-\infty$; with $c$ we denote generic constants.

Note first that if $\lim V(s)=P_{2}$ then the solution is weakly singular.

In order to prove the converse, we claim that

$$
v^{\prime}(s) \rightarrow-4
$$

To this end, we exclude all the other cases; recall that $\lim v^{\prime}(s)$ exists by definition of weakly critical solution.

(A) It cannot be $\lim v^{\prime}(s)=c \in(-\infty,-4)$.

For contradiction, if $\lim v^{\prime}(s)=c<-4$, then by $(7)_{1}$ we infer

$$
\lim v_{1}(s)=c+4<0
$$

and by $(7)_{4}$ we get

$$
v_{4}(s) \rightarrow-\infty
$$

Write $(8)_{3}$ as

$$
\frac{d}{d s}\left[e^{(n-4) s} v_{3}(s)\right]=e^{(n-4) s} v_{4}(s)
$$

so that by (16) we infer that the map $s \mapsto e^{(n-4) s} v_{3}(s)$ is decreasing in a neighbourhood of $-\infty$ and therefore it admits a limit. If $e^{(n-4) s} v_{3}(s) \rightarrow c \geq 0$ then by $(8)_{3}$ and (16) we get $v_{3}^{\prime}(s) \rightarrow-\infty$ and hence $v_{3}(s) \rightarrow+\infty$. If $e^{(n-4) s} v_{3}(s) \rightarrow c<0$ then $v_{3}(s) \rightarrow-\infty$. In any case we obtain

$$
\left|v_{3}(s)\right| \rightarrow+\infty
$$

A completely similar (but slightly more involved) argument shows that $(8)_{2}$ and (17) entail

$$
\left|v_{2}(s)\right| \rightarrow+\infty
$$


Finally, (8) 1 (15) and (18) furnish $\left|v_{1}^{\prime}(s)\right| \rightarrow+\infty$ which contradicts (15).

(B) It cannot be $v^{\prime}(s) \rightarrow-\infty$.

For contradiction, assume that $v^{\prime}(s) \rightarrow-\infty$ : then by $(7)_{1}$ we have

$$
v_{1}(s) \rightarrow-\infty
$$

and by $(7)_{4}$ we get

$$
v_{4}(s) \rightarrow-\infty
$$

moreover,

$$
\frac{v(s)}{s} \rightarrow-\infty
$$

We may rewrite $(8)_{3}$ as

$$
\frac{d}{d s}\left[e^{(n-4) s} v_{3}(s)\right]=e^{(n-4) s} v_{4}(s)=-\lambda e^{n s+v(s)} \rightarrow-\infty,
$$

where the second equality is just $(7)_{4}$ and the infinite limit is a consequence of (21): the previous limit yields $e^{(n-4) s} v_{3}(s) \rightarrow+\infty$ and, in turn,

$$
v_{3}(s) \rightarrow+\infty
$$

Similarly, we may rewrite $(8)_{2}$ as

$$
\frac{d}{d s}\left[e^{-2 s} v_{2}(s)\right]=e^{-2 s} v_{3}(s) \rightarrow+\infty
$$

where the infinite limit is a consequence of (22): hence, we deduce that $e^{-2 s} v_{2}(s) \rightarrow-\infty$ which, together with $(8)_{2}$ and $(22)$, shows that $v_{2}(s) \rightarrow-\infty$. Inserting this into $(7)_{2}$ gives $v^{\prime \prime}(s)+(n-2) v^{\prime}(s) \rightarrow+\infty$ and therefore $v^{\prime}(s)+(n-2) v(s) \rightarrow-\infty$ : hence,

$$
\text { there exists } \sigma<0 \quad \text { such that } \quad v^{\prime}(s)+(n-2) v(s)<0 \quad \text { for all } s \leq \sigma \text {. }
$$

We rewrite this inequality as

$$
\frac{d}{d s}\left[e^{(n-2) s} v(s)\right]<0 \quad \text { for all } s \leq \sigma
$$

integrating it over $[s, \sigma]$ and taking into account that $v(\sigma)>0$, we infer that

$$
\text { there exists } K>0 \quad \text { such that } v(s) \geq K e^{(2-n) s} \quad \text { for all } s \leq \sigma .
$$

Using (5) and returning to the function $u$ (solution of $\left(P_{\lambda}\right)$ and $(4)$ ), this shows that

$$
\text { there exists } K>0 \quad \text { such that } u(r) \geq \frac{K}{r^{n-2}} \quad \text { for all } r \leq e^{\sigma} \text {; }
$$

this contradicts $e^{u} \in L^{1}(B)$.

(C) It cannot be $\lim v^{\prime}(s)=c \in(-4,0]$.

For contradiction, if $\lim v^{\prime}(s)=c \in(-4,0]$, then by $(7)_{1}$ we infer

$$
\lim v_{1}(s)=c+4>0
$$


and by $(7)_{4}$ we get

$$
v_{4}(s) \rightarrow 0
$$

Then, from $(8)_{3}$ we deduce

$$
v_{3}(s) \rightarrow 0
$$

because otherwise, we would get a similar contradiction as in Case (A). Next, from (8) 2 and (25) we obtain

$$
v_{2}(s) \rightarrow 0 \text {. }
$$

Since by assumption, $v_{1}$ has a limit, we deduce that necessarily $v_{1}(s) \rightarrow 4$. This, together with (24) (25) and (26) contradicts part $(i)$ proved above.

By $(\mathrm{A})(\mathrm{B})(\mathrm{C})$, the statement $(14)$ is proved. This shows that $v_{1}(s) \rightarrow 0$ : inserting this into $(8)_{1}$ gives $v_{2}(s) \rightarrow 4(n-2)$. Inserting the latter into $(8)_{2}$ yields $v_{3}(s) \rightarrow-8(n-2)$; finally, inserting this into $(8)_{3}$ gives $v_{4}(s) \rightarrow-8(n-2)(n-4)$. This completes the proof of (ii).

Remark 5. If in case $(\mathrm{ii})$ of Theorem 3 we do not assume that $\lim v^{\prime}(s)$ exists, then we can merely show that $\liminf v^{\prime}(s) \leq-4 \leq \lim \sup v^{\prime}(s)$. Clearly, if one could prove that both inequalities are in fact equalities, then we would have again (14).

\section{Proof of Theorem 1}

We denote by $\mathcal{K}$ the cone of nonnegative $L^{2}$-functions in $B$,

$$
\mathcal{K}=\left\{u \in L^{2}(B) ; u(x) \geq 0 \text { for a.e. } x \in B\right\}
$$

and (for the sake of completeness) we prove a weak formulation of Boggio's positivity preserving property [B], which we extensively use:

Lemma 1. Assume that $u \in L^{2}(B)$ satisfies

$$
\int_{B} u \Delta^{2} v \geq 0 \quad \text { for all } v \in \mathcal{K} \cap H^{4} \cap H_{0}^{2}(B)
$$

then $u \in \mathcal{K}$. Moreover, one has either $u \equiv 0$ or $u>0$ a.e. in $B$.

Proof. (i) Take any $\varphi \in \mathcal{K} \cap C_{c}^{\infty}(B)$ and let $v_{\varphi}$ be the unique (classical) solution of

$$
\left\{\begin{array}{l}
\Delta^{2} v_{\varphi}=\varphi \text { in } B \\
v_{\varphi}=\frac{\partial v_{\varphi}}{\partial \mathbf{n}}=0 \text { on } \partial B .
\end{array}\right.
$$

Then, by the classical Boggio's principle [B] we infer that $v_{\varphi} \in \mathcal{K}$. Hence, $v_{\varphi}$ is a possible test function for all so chosen $\varphi$ and therefore

$$
\int_{B} u \varphi=\int_{B} u \Delta^{2} v_{\varphi} \geq 0 \quad \text { for all } \varphi \in \mathcal{K} \cap C_{c}^{\infty}(B) .
$$

This shows that $u \in \mathcal{K}$. 
(ii) By $(i)$ we know that $u \in \mathcal{K}$. So, assume that $u \ngtr 0$ a.e. in $B$ and let $\phi$ denote the characteristic function of the set $\{x \in B ; u(x)=0\}$ so that $\phi \geq 0, \phi \not \equiv 0$. Let $v_{0}$ be the unique (a.e.) solution of the problem

$$
\left\{\begin{array}{l}
\Delta^{2} v_{0}=\phi \quad \text { in } B \\
v_{0}=\frac{\partial v_{0}}{\partial \mathbf{n}}=0 \text { on } \partial B
\end{array}\right.
$$

Then,

$$
v_{0} \in\left(\bigcap_{q \geq 1} W^{4, q}(B)\right) \subset C^{3}(\bar{B})
$$

and by Boggio's principle [B] we have $v_{0}>0$ in $B$. By the biharmonic analogue of Hopf's Lemma in balls (see [GS, Theorem 3.2] which also holds if $\Delta^{2} v_{0} \in L^{p}(B)$ for some $p>n / 2$ ) we necessarily have $\Delta v_{0}>0$ on $\partial B$. This last inequality allows us to state that for all $v \in C^{4}(\bar{B}) \cap H_{0}^{2}(B)$ there exists $t_{1} \leq 0 \leq t_{0}$ such that $v+t_{0} v_{0} \geq 0$ and $v+t_{1} v_{0} \leq 0$ in $B$. This, combined with the fact that

$$
\int_{B} u \Delta^{2} v_{0}=\int_{\{u=0\}} u=0
$$

enables us to show that both

$$
0 \leq \int_{B} u \Delta^{2}\left(v+t_{0} v_{0}\right)=\int_{B} u \Delta^{2} v \quad \text { and } \quad 0 \geq \int_{B} u \Delta^{2}\left(v+t_{1} v_{0}\right)=\int_{B} u \Delta^{2} v .
$$

Hence, we have for all $v \in C^{4}(\bar{B}) \cap H_{0}^{2}(B)$

$$
\int_{B} u \Delta^{2} v=0
$$

We need to show that $C^{4}(\bar{B}) \cap H_{0}^{2}(B)$ is dense in $H^{4} \cap H_{0}^{2}(B)$. For this purpose, take any function $U \in H^{4}(B) \cap H_{0}^{2}(B)$ and put $f:=\Delta^{2} U$. We approximate $f$ in $L^{2}(B)$ by $C^{\infty}(\bar{B})-$ functions $f_{k}$ and solve $\Delta^{2} U_{k}=f_{k}$ in $B$ under homogeneous Dirichlet boundary conditions. We then even have $U_{k} \in C^{\infty}(\bar{B})$, and by $L^{2}$-theory there holds $\left\|U_{k}-U\right\|_{H^{4}(B)} \rightarrow 0$ as $k \rightarrow \infty$.

By the previous statement we may now conclude:

$$
\text { for all } v \in H^{4} \cap H_{0}^{2}(B): \quad \int_{B} u \Delta^{2} v=0 .
$$

Since $u \in L^{2}(\Omega)$, we may take as $v \in H^{4} \cap H_{0}^{2}(B)$ the solution of $\Delta^{2} v=u$ under homogeneous Dirichlet boundary conditions. This finally yields $u \equiv 0$.

In particular, thanks to Lemma 1 we may establish a result in the spirit of [BCMR]:

Lemma 2. For all $f \in L^{1}(B)$ such that $f \geq 0$ a.e. in $B$ there exists a unique $u \in L^{1}(B)$ such that $u \geq 0$ a.e. in $B$ and which satisfies

$$
\int_{B} u \Delta^{2} v=\int_{B} f v \quad \text { for all } v \in C^{4}(\bar{B}) \cap H_{0}^{2}(B) ;
$$

moreover, there exists $C>0$ (independent of $f$ ) such that $\|u\|_{1} \leq C\|f\|_{1}$. 
Proof. Uniqueness follows by means of the observation that $L^{\infty}$-functions may be approximated by a pointwise convergent but uniformly bounded sequence of $C_{c}^{\infty}(B)$-functions. This is applied to truncations of $u$ and suitable test functions $v$ are obtained from approximations of the truncations of $u$ by solving the biharmonic Dirichlet problems.

Existence follows by truncating $f$ and by arguing as in the proof of [BCMR, Lemma 1], the only difference being the positivity preserving property which is standard for the Laplacian: in our case we invoke Lemma 1.

Combining the method of proof of Lemmas 1 and 2, one also has

Lemma 3. Assume that $u \in L^{1}(B)$ satisfies

$$
\int_{B} u \Delta^{2} v d x \geq 0 \quad \text { for all } v \in \mathcal{K} \cap C^{4}(\bar{B}) \cap H_{0}^{2}(B),
$$

then $u \geq 0$ a.e. in $B$.

The previous lemmas enable us to make use of the super-subsolutions method:

Lemma 4. Let $\lambda>0$ and assume that there exists $\bar{u} \in \mathcal{K}$ such that $e^{\bar{u}} \in L^{1}(B)$ and

$$
\int_{B} \bar{u} \Delta^{2} v \geq \lambda \int_{B} e^{\bar{u}} v \quad \text { for all } v \in \mathcal{K} \cap W^{4, p} \cap H_{0}^{2}(B) .
$$

Then there exists a solution $u$ of $\left(P_{\lambda}\right)$ such that $0 \leq u \leq \bar{u}$ a.e. in $B$.

Proof. Let $u_{0}=\bar{u}$ and for all $m \in \mathbb{N}$, define inductively the function $u_{m+1}$ as the unique solution of

$$
\int_{B} u_{m+1} \Delta^{2} v=\lambda \int_{B} e^{u_{m}} v \quad \text { for all } v \in W^{4, p} \cap H_{0}^{2}(B) .
$$

Note that by Lemmas 1 to 3 the sequence $\left\{u_{m}\right\}$ is well-defined and

$$
u_{m} \in \mathcal{K}, \quad e^{u_{m}} \in L^{1}(B), \quad 0 \leq u_{m+1}(x) \leq u_{m}(x) \text { for a.e. } x \in B \quad \text { for all } m \in \mathbb{N} \text {. }
$$

Since this sequence is pointwise decreasing, there exists $u \in \mathcal{K}$ such that $e^{u} \in L^{1}(B)$ and which is the pointwise limit of $\left\{u_{m}\right\}$. Then, letting $m \rightarrow \infty$ in (27) and applying Lebesgue's Theorem, we obtain the result.

Define $\Lambda:=\left\{\lambda \geq 0 ;\left(P_{\lambda}\right)\right.$ admits a solution $\}$ and

$$
\lambda^{*}:=\sup \Lambda
$$

clearly $0 \in \Lambda$ and so $\Lambda \neq \emptyset$. Moreover, by the implicit function theorem we know that $\lambda^{*}>0$. It follows directly from Lemma 4 that $\Lambda$ is an interval.

Let $\lambda \in \Lambda$; then, there exists $u_{\lambda}$ satisfying (2). Taking into account that $e^{s} \geq e s$ for all $s \geq 0$ with strict inequality whenever $s \neq 1$, and choosing $v=\phi_{1}$ (the normalized positive first eigenfunction of (3)) as test function in (2), we get

$$
\lambda_{1} \int_{B} u_{\lambda} \phi_{1}=\int_{B} u_{\lambda} \Delta^{2} \phi_{1}=\lambda \int_{B} e^{u_{\lambda}} \phi_{1}>\lambda e \int_{B} u_{\lambda} \phi_{1}
$$


which proves that

$$
\lambda<\frac{\lambda_{1}}{e} \quad \text { for all } \lambda \in \Lambda \text {. }
$$

We now prove the most delicate part of Theorem 1, namely that for any $\lambda<\lambda^{*}$, there exists a regular solution:

Lemma 5. Assume that for some $\mu>0$ there exists a (possibly singular) solution $u_{0}$ of $\left(P_{\mu}\right)$. Then, for all $0<\lambda<\mu$ there exists a regular solution of $\left(P_{\lambda}\right)$.

Proof. Let $0<\lambda<\mu$ and consider the (unique) functions $u_{1}, u_{2} \in L^{1}(B)$ satisfying respectively

$$
\begin{array}{ll}
\int_{B} u_{1} \Delta^{2} v=\lambda \int_{B} e^{u_{0}} v & \text { for all } v \in W^{4, p} \cap H_{0}^{2}(B) \\
\int_{B} u_{2} \Delta^{2} v=\lambda \int_{B} e^{u_{1}} v & \text { for all } v \in W^{4, p} \cap H_{0}^{2}(B) .
\end{array}
$$

Such functions exist by Lemma 2 and also belong to $L^{2}(B)$ since by Lemma 3 we have

$$
u_{0}>\frac{\lambda}{\mu} u_{0}=u_{1} \geq u_{2} \quad \text { a.e. in } B \text {. }
$$

Let $\varphi(x)=\left(1-|x|^{2}\right)^{2}$; it is readily verified that

$$
\varphi \in H_{0}^{2}(B), \quad \Delta^{2} \varphi=8 n(n+2) .
$$

We also need the following elementary statement:

for all $\vartheta>1$ and $\delta>0$ there exists $\gamma>0$ such that $e^{\vartheta s}+\gamma-(1+\delta) e^{s} \geq 0$ for all $s \geq 0$.

Take $\vartheta=\mu / \lambda, \delta=n \lambda / 4 \mu$ and choose $k>0$ in such a way that

$$
e^{\frac{\mu}{\lambda} s}+\frac{8 n(n+2)}{\lambda} k \geq(1+\delta) e^{s} \quad \text { for all } s \geq 0 ;
$$

this choice is clearly allowed by (32). Thanks to (31) and (33) we find

$$
\begin{gathered}
\int_{B}\left(u_{1}+k \varphi\right) \Delta^{2} v=\int_{B}\left[\lambda e^{u_{0}}+8 n(n+2) k\right] v= \\
\int_{B}\left[\lambda e^{\frac{\mu}{\lambda} u_{1}}+8 n(n+2) k\right] v \geq \lambda(1+\delta) \int_{B} e^{u_{1}} v=(1+\delta) \int_{B} u_{2} \Delta^{2} v \quad \text { for all } v \in \mathcal{K} \cap W^{4, p} \cap H_{0}^{2}(B) .
\end{gathered}
$$

Hence, by Lemma 1 we infer that $u_{2} \leq \frac{u_{1}+k \varphi}{1+\delta}$ in $B$; in particular, we get

$$
e^{u_{2}} \leq e^{\frac{k}{1+\delta} \varphi} e^{\frac{\lambda}{\mu(1+\delta)} u_{0}}
$$

from which we get at once that

$$
e^{u_{2}} \in L^{\frac{n}{4}+\frac{\mu}{\lambda}}(B)
$$

since $\varphi \in L^{\infty}(B)$ and $e^{u_{0}} \in L^{1}(B)$ (recall also our choice of $\delta$ ). Finally, consider $u_{3} \in L^{2}(B)$ such that

$$
\int_{B} u_{3} \Delta^{2} v=\lambda \int_{B} e^{u_{2}} v \quad \text { for all } v \in W^{4, p} \cap H_{0}^{2}(B) .
$$


By (34) and elliptic regularity [ADN], we deduce that

$$
u_{3} \in W^{4, \frac{n}{4}+\frac{\mu}{\lambda}}(B) \subset L^{\infty}(B) .
$$

Moreover, by (29), (30) and Lemma 1 we infer that $u_{3} \leq u_{2}$ and hence

$$
\int_{B} u_{3} \Delta^{2} v \geq \lambda \int_{B} e^{u_{3}} v \quad \text { for all } v \in \mathcal{K} \cap W^{4, p} \cap H_{0}^{2}(B) .
$$

We have so found a weak bounded supersolution $u_{3}$ of $\left(P_{\lambda}\right)$ and the statement follows from Lemma 4.

With help of Lemma 5 we can now show:

Lemma 6. For all $0 \leq \lambda<\lambda^{*}$, the minimal solution $U_{\lambda}$ exists, is regular and radially symmetric.

Proof. By the preceding lemma we have existence of a regular solution $u_{\lambda}$ of $\left(P_{\lambda}\right)$. This may serve as a (classical) supersolution of $\left(P_{\lambda}\right)$ while $U_{0} \equiv 0$ is a subsolution. Hence, the minimal solution $U_{\lambda}$ of $\left(P_{\lambda}\right)$ may be obtained as the increasing limit of the following sequence $\left\{U_{m}\right\}$ :

$$
\left\{\begin{array}{ll}
\Delta^{2} U_{m+1}=\lambda e^{U_{m}} & \text { in } B \\
U_{m+1}=\frac{\partial U_{m+1}}{\partial \mathbf{n}}=0 & \text { on } \partial B
\end{array} \quad(m \geq 0) .\right.
$$

Since $U_{0}$ is radially symmetric, so is $U_{1}$; similarly, all the functions $U_{m}$ are radially symmetric: therefore, their (pointwise) limit $U_{\lambda}$ is also radially symmetric.

The previous lemma allows us to show that the interval $\Lambda$ is closed: we first remark that the map $\lambda \mapsto U_{\lambda}(x)$ is strictly increasing for all $x \in B$ (in view of Lemma 1). If $0 \leq \lambda<\mu<\lambda^{*}$, the minimal solution $U_{\mu}$ of $\left(P_{\mu}\right)$ is a (strict) supersolution for $\left(P_{\lambda}\right)$. Therefore

$$
U_{*}(x):=\lim _{\lambda \rightarrow \lambda^{*}} U_{\lambda}(x) \in[0, \infty]
$$

exists for all $x \in B$. In fact, more can be said about this limit:

Lemma 7. Let $U_{*}$ be the function defined in (35). Then $U_{*}(x)$ is finite for a.e. $x \in B$ and $U_{*}$ solves $\left(P_{\lambda}\right)$ for $\lambda=\lambda^{*}$. Moreover, $U_{\lambda} \rightarrow U_{*}$ in $H_{0}^{2}(B)$ as $\lambda \uparrow \lambda^{*}$. Finally, $U_{*}$ is radially symmetric.

Proof. By Lemma 6 we have $U_{\lambda} \in C^{\infty}(\bar{B})$ and therefore, by using the generalized Pohozaev identity $[\mathrm{P}]$ by Pucci-Serrin [PS] and by arguing as in the proof of [GMP, Théorème 2] we obtain that the set $\left\{U_{\lambda} ; \lambda<\lambda^{*}\right\}$ is bounded in $H_{0}^{2}(B)$, hence $U_{\lambda} \rightarrow U_{*}$ in $H_{0}^{2}(B)$, up to a subsequence (this follows by uniqueness of the pointwise limit). This shows that $U_{*}$ is a.e. finite, that $U_{*}$ solves $\left(P_{\lambda}\right)$ for $\lambda=\lambda^{*}$ and also that $U_{*} e^{U_{*}} \in L^{1}(B)$. Finally, since $U_{\lambda} e^{U_{\lambda}} \leq U_{*} e^{U_{*}}$, by Lebesgue's Theorem we deduce that

$$
\int_{B}\left|\Delta U_{\lambda}\right|^{2}=\int_{B} U_{\lambda} e^{U_{\lambda}} \rightarrow \int_{B} U_{*} e^{U_{*}}=\int_{B}\left|\Delta U_{*}\right|^{2} \quad \text { as } \lambda \uparrow \lambda^{*}
$$


which, together with weak convergence, shows that $U_{\lambda} \rightarrow U_{*}$ in the norm topology of $H_{0}^{2}(B)$; since the above arguments may be repeated for any sequence in $\left\{U_{\lambda} ; \lambda<\lambda^{*}\right\}$, the result follows without extracting subsequences.

Finally, by Lemma 6 , all the minimal solutions $U_{\lambda}$ (for $0<\lambda<\lambda^{*}$ ) are radially symmetric. Then, by (35) also $U_{*}$ is radially symmetric.

Remark 6. The proof of Lemma 7 may also be obtained by exploiting the stability of the minimal solution $U_{\lambda}$ (see Proposition $4(i)$ below) and by arguing as in [BV, Remark 3.3].

Finally, we claim that

$$
\lambda^{*} \geq 14.72(n-1)(n-3) .
$$

Indeed, this holds true by Lemma 4 since the function $\bar{u}(x)=7.36(1-|x|)^{2}$ is a weak supersolution $\left(\bar{u} \in C^{\infty}(\bar{B} \backslash\{0\})\right)$ of $\left(P_{\lambda}\right)$ for all $\lambda \leq 14.72(n-1)(n-3)$.

Proof of Theorem 1. The upper bound for $\lambda^{*}$ follows from (28) and from Lemma 7, the latter saying that $\lambda^{*} \in \Lambda$. The lower bound for $\lambda^{*}$ is proved in (36). Statement $(i)$ follows from Lemmas 5 and 6 . The map $\lambda \mapsto U_{\lambda}(x)$ is non-decreasing for all $x$ by Lemma 4 and strictly increasing by Lemma 1 ; this proves the first part of statement $(i i)$. The second part of $(i i)$ and (iii) follow from Lemma 7. Finally, the radial symmetry of $U_{*}$ and of all the minimal solutions $U_{\lambda}$ (for $\lambda<\lambda^{*}$ ) is obtained in Lemmas 7 and 6 respectively. The regular minimal solutions $U_{\lambda}$ (for $\lambda<\lambda^{*}$ ) are strictly radially decreasing in view of [So]. Passing to the limit, we also get that $U^{*}$ is radially decreasing.

Remark 7. The above analysis does not allow us to establish whether the extremal solution $U_{*}$ is regular, weakly singular or singular. However, since it is radially symmetric, in the first two cases Theorem 3 below describes the behaviour of $U_{*}$ when studied in the phase space $\mathbb{R}^{4}$. With our computer assisted proof, we may then find some space dimensions where the first case certainly occurs, provided that we can also show uniqueness of the weakly singular solution and the corresponding parameter $\lambda_{\sigma}$.

\section{Proof of Theorem 2}

We first show that

$$
U_{\lambda} \rightarrow 0 \quad \text { uniformly as } \lambda \rightarrow 0 .
$$

Since this is standard, we just briefly sketch its proof. By Theorem 1 we know that

$$
0<\lambda<\mu<\lambda^{*} \Longrightarrow U_{\lambda}(x)<U_{\mu}(x) \quad \text { if }|x|<1 .
$$

Then, by multiplying the equation in $\left(P_{\lambda}\right)$ by $U_{\lambda}$ and by integrating by parts we obtain that $\left\|U_{\lambda}\right\|_{H_{0}^{2}(B)}$ remains bounded. Hence, up to a subsequence, $\left\{U_{\lambda}\right\}$ converges in the weak $H_{0}^{2}(B)$ topology to $U_{0} \equiv 0$, which is the unique solution of $\left(P_{0}\right)$. By convergence of the norms, we infer that the convergence is in the norm topology. Finally, by pointwise convergence and elliptic regularity we infer (37).

Next, note that $V_{\lambda}$ satisfies

$$
\begin{cases}\Delta^{2} V_{\lambda}=\lambda & \text { in } B \\ V_{\lambda}=\frac{\partial V_{\lambda}}{\partial \mathbf{n}}=0 & \text { on } \partial B\end{cases}
$$


Therefore, $\Delta^{2} U_{\lambda}>\Delta^{2} V_{\lambda}$ and the inequality $U_{\lambda}>V_{\lambda}$ follows by Lemma 1 .

In order to prove the last statement of Theorem 2, note that from (37) we infer

$$
\text { for all } \varepsilon>0 \text { there exists } \lambda_{\varepsilon}>0 \text { such that } \quad \lambda<\lambda_{\varepsilon} \Longrightarrow\left\|U_{\lambda}\right\|_{\infty}<\varepsilon \text {. }
$$

So, fix $\varepsilon>0$ and let $\lambda<\lambda_{\varepsilon}$. Then, (38) entails

$$
\Delta^{2} U_{\lambda}=\lambda e^{U_{\lambda}}<\lambda e^{\varepsilon}=e^{\varepsilon} \Delta^{2} V_{\lambda} \quad \text { in } B .
$$

This shows that $U_{\lambda}(x)<e^{\varepsilon} V_{\lambda}(x)$ for all $x \in B$ and the result follows by arbitrariness of $\varepsilon$.

\section{Proof of Theorem 4}

The proof of Theorem 4 is obtained with computer assistance. We first describe the numerical procedure used to obtain the approximate values for $\lambda_{\sigma}$ and $\lambda^{*}$, then we show how the algorithm can be made rigorous. We maintain here the same notations as in Section 3 . The computation of $\lambda_{\sigma}$ is somehow simpler than the computation of $\lambda^{*}$, since the unstable manifold of $P_{2}$ is one-dimensional. We choose a point $\bar{v}=P_{2}+r e_{1}$ where $e_{1}$ is an eigenvector corresponding to the unstable manifold and $r$ is some small value. We solve the system (8) with $\bar{v}$ as initial condition and we look for the intersection of the solution with the hyperplane $v_{1}=4$. The choice of a positive or negative $r$ leads to different results, since the manifold is made of two branches: it turns out that one branch never appears to intersect the hyperplane, while the other branch always does. Since we are proving the existence of one solution, we can neglect the first branch and focus on the second one. If the solution intersects the hyperplane $v_{1}=4$ at some point $\hat{v}=\left(\hat{v}_{1}, \hat{v}_{2}, \hat{v}_{3}, \hat{v}_{4}\right)$ such that $\hat{v}_{4}<0$, by Theorem 3 and by equation (7) we have numerical evidence of a singular solution at $\lambda=-\hat{v}_{4}$.

In order to compute the value of $\lambda^{*}$ we have to study the two-dimensional unstable manifold of $P_{1}$. The direction on the tangent hyperplane can be parametrized by an angle $\vartheta$. In order to find the largest value for $\lambda$ we use a directional shooting method, i.e. we choose some value $\vartheta$ (the shooting direction) and solve the equation with starting point $\bar{v}=P_{1}+r\left(e_{1} \sin \vartheta+\right.$ $e_{2} \cos \vartheta$ ), where $e_{1}$ and $e_{2}$ are the orthonormalized eigenvectors corresponding to the (tangent) unstable manifold and $r>0$ is some small arbitrarily chosen value. If the solution intersects the hyperplane $v_{1}=4$ at some point $\hat{v}=\left(\hat{v}_{1}, \hat{v}_{2}, \hat{v}_{3}, \hat{v}_{4}\right)$ such that $\hat{v}_{4}<0$, then by Theorem 3 and by equations (7) and (8) we have numerical evidence of a regular solution for $\lambda=-\hat{v}_{4}$. By varying $\vartheta$ we can look for the maximal value of $\lambda$.

Of course these procedures do not lead to an exact value for two reasons. First, we can only choose $\bar{v}$ on the unstable manifold of the linearized equation, and although we know that we are close to the manifold of the full equation, we are not exactly on it. Second, the algorithm used to solve the differential equation provides an accurate, but not rigorous, solution. We address the problem of proving that a branch of the unstable manifold of $P_{2}$ does intersect the hyperplane $v_{1}=4$ and of computing a rigorous estimate for the values $\lambda_{\sigma}$ and $\lambda^{*}$ in the next sections.

\subsection{Rigorous bounds for the manifolds}

We first address the general problem of computing rigorous bounds for the location of the unstable manifold in the neighbourhood of a stationary hyperbolic point of an ordinary 
differential equation. The same technique could be applied to the stable manifold as well, but in this paper we are not interested in it.

Let $f \in C^{2}\left(\mathbb{R}^{d}, \mathbb{R}^{d}\right), d \geq 2$. We consider the equation $\dot{x}=f(x)$ and we assume that 0 is a hyperbolic stationary point. Then

$$
\dot{x}=A x+N(x),
$$

where

$$
A=\nabla f(0), N(x)=O\left(|x|^{2}\right) \text { as } x \rightarrow 0
$$

and all eigenvalues of $A$ have nonzero real part. Let $\varphi(x, t)$ be the flow induced by equation (39) and let $\varphi_{A}(x, t)$ be the flow induced by the linear equation $\dot{x}=A x$. Let $S_{0}$ (resp. $U_{0}$ ) be the span of all eigenvectors corresponding to the eigenvalues with negative (resp. positive) real part. $S_{0}$ (resp. $U_{0}$ ) is called the stable (resp. unstable) subspace, and it is characterized as follows: $S_{0}$ (resp. $U_{0}$ ) is the set of points $x \in \mathbb{R}^{d}$ such that $\varphi_{A}(x, t) \rightarrow 0$ as $t \rightarrow+\infty$ (resp. $t \rightarrow-\infty$ ). It is well known that the full equation also admits a stable manifold $S$ (resp. an unstable manifold $U$ ) still defined as the set of points $x \in \mathbb{R}^{d}$ such that $\varphi(x, t) \rightarrow 0$ as $t \rightarrow+\infty$ (resp. $t \rightarrow-\infty$ ). Such manifold is tangent at the origin to $S_{0}$ (resp. $U_{0}$ ). If $S_{0}$ (resp. $U_{0}$ ) is empty, then there exists a neighbourhood of the origin which is a subset of $U$ (resp. $S$ ). We are interested in the case when both manifolds are nontrivial and we wish to study the intersection of the unstable manifold with some other manifold $P$. In order to achieve this goal, we consider a point $\bar{x} \in U \backslash\{0\}$ and study $\varphi(\bar{x}, t)$. If we can prove that $\varphi\left(\bar{x}, t_{0}\right) \in P$ for some positive $t_{0}$, then we infer that $U \cap P \neq \emptyset$ and we also know the intersection point. The main problem to address is that the only point of the manifold we know precisely is the origin: the other points lie very close to $U_{0}$, at least in a neighbourhood of 0 , but we do not know their explicit position. We proceed as follows.

There exists an invertible matrix $M$ such that $B:=M^{-1} A M$ is block diagonal, i.e. the canonical basis $\left\{e_{i}\right\}$ of $\mathbb{R}^{d}$ is split in $S_{0}^{\prime} \cup U_{0}^{\prime}$, where $S_{0}^{\prime}=\operatorname{span}\left\{e_{1}, \ldots, e_{m}\right\}$ is the stable eigenspace and $U_{0}^{\prime}=\operatorname{span}\left\{e_{m+1}, \ldots, e_{d}\right\}$ is the unstable eigenspace. If we let $y=M^{-1} x$ equation (39) writes

$$
\dot{y}=B y+M^{-1} N(M y)=: g(y) .
$$

By (40), for all $\varepsilon>0$ there exists $\beta>0$ such that $|N(x)| \leq \beta|x|^{2}$ for all $|x| \leq \varepsilon$. Let $\alpha<0$ be the maximum of the real parts of the eigenvalues with negative real parts, $\gamma=-\frac{\alpha}{\beta m_{1}^{2} m_{2}}$, $m_{1}=\|M\|$ and $m_{2}=\left\|M^{-1}\right\|$. Choose $\varepsilon>0$, let $\beta>0$ as above, choose a vector $\hat{y} \in U_{0}^{\prime} \backslash\{0\}$ of norm $r \leq \varepsilon$ and choose $k>1$. Let $P_{s}$ be the orthogonal projection onto $S_{0}^{\prime}$, let $P_{u}$ be the orthogonal projection onto the linear space spanned by $\hat{y}$ and let

$$
\Xi=\left\{y \in \mathbb{R}^{d}: \frac{\gamma}{k}\left|P_{s} y\right| \leq\left|P_{u} y\right|^{2} \leq r^{2}\right\} .
$$

We show that, under a suitable choice of $k>1$ and $0<r \leq \varepsilon$, for all $y \in \partial \Xi$ such that $\left|P_{u} y\right|<r$ the flow is inward, i.e. given $\bar{y} \in \Xi$ we want the solution of the Cauchy problem $\dot{y}(t)=g(y(t)), y(0)=\bar{y}$ to leave $\Xi$ only through the set $\left\{y \in \partial \Xi:\left|P_{u} y\right|=r\right\}$. If this happens, then for all $\hat{y} \in U_{0}^{\prime}$ satisfying $|\hat{y}|=r$ either the unstable manifold intersects the set

$$
\kappa:=\kappa_{\hat{y}}:=\hat{y}+\left\{\tilde{y} \in S_{0}^{\prime}:|\tilde{y}| \leq \frac{k}{\gamma} r^{2}\right\},
$$

or it is entirely contained in $\Xi$. As a result, to study a branch of the unstable manifold it is sufficient to exclude the second case and consider the initial value problem for all $\bar{y} \in \kappa$. 
Lemma 8. Choose $\varepsilon>0$ and $k>1$. Let $\alpha, \beta, \gamma, m_{1}, m_{2}$ and $\Xi$ be as above and let

$$
r=\min \left\{\frac{\varepsilon \gamma}{m_{1} \sqrt{\gamma^{2}+k^{2}}}, 1, \frac{\sqrt{k-1}}{k} \gamma, \frac{\gamma}{2}\right\} .
$$

For all $\bar{y} \in \partial \Xi$ such that $0<\left|P_{u} \bar{y}\right|<r$ we have

$$
\left(g(\bar{y}), P_{s} \bar{y}\right)<0 .
$$

Proof. Let $\hat{y}=P_{s} \bar{y}, \tilde{y}=P_{u} \bar{y}, \hat{r}=|\hat{y}|$ and $\tilde{r}=|\tilde{y}|$. Since $\tilde{r}<r \leq \frac{\varepsilon \gamma}{m_{1} \sqrt{\gamma^{2}+k^{2}}}$, then $|M \bar{y}| \leq \varepsilon$ and therefore $|N(\bar{y})| \leq \beta|\bar{y}|^{2}$. We have

$$
\begin{gathered}
(B \bar{y}, \hat{y})+\left(M^{-1} N(M \bar{y}), \hat{y}\right)=(B \hat{y}, \hat{y})+\left(N(M \bar{y}),\left(M^{-1}\right)^{t} \hat{y}\right) \\
\leq \alpha \hat{r}^{2}+\beta m_{1}^{2} m_{2}\left(\hat{r}^{2}+\tilde{r}^{2}\right) \hat{r}
\end{gathered}
$$

Then, a simple computation shows that (45) is implied by

$$
\frac{1}{2}\left(\gamma+\sqrt{\gamma^{2}-4 \tilde{r}^{2}}\right)>\hat{r}>\frac{1}{2}\left(\gamma-\sqrt{\gamma^{2}-4 \tilde{r}^{2}}\right) .
$$

The first inequality is satisfied because $\hat{r}<r \leq \frac{\gamma}{2}$. To show the second one, note that for all $\bar{y} \in \partial \Xi, 0<\left|P_{u} \bar{y}\right|<r$ by (42) we have $\hat{r}=\frac{k}{\gamma} \tilde{r}^{2}$ and

$$
\frac{1}{2}\left(\gamma-\sqrt{\gamma^{2}-4 \tilde{r}^{2}}\right)<\frac{k}{\gamma} \tilde{r}^{2} \quad \text { if } \quad \tilde{r}<\frac{\sqrt{k-1}}{k} \gamma .
$$

Since $\tilde{r}<r$, then (46), and subsequently (45) hold.

We need a condition which ensures that the invariant manifold is not entirely contained in $\Xi$, but it intersects $\kappa$ at some point. Let $\alpha^{\prime}$ be the minimum of the real parts of the eigenvalues of $B$ with positive real parts.

Lemma 9. If $r, \alpha, \alpha^{\prime}, k$ and $\Xi$ are as above and

$$
\alpha^{\prime}+\frac{\alpha}{\gamma}\left(r+\frac{k^{2}}{\gamma^{2}} r^{3}\right)>0
$$

then there exists $\delta>0$ such that $\left(g(\bar{y}), P_{u} \bar{y}\right) \geq \delta\left|P_{u} \bar{y}\right|^{2}$ for all $\bar{y} \in \Xi$ and therefore the component of the flow in the direction of the unstable manifold is always increasing in $\Xi$, together with its first derivative.

Proof. Choose $\delta>0$ satisfying

$$
\beta m_{1}^{2} m_{2}\left(r+\frac{k^{2}}{\gamma^{2}} r^{3}\right) \leq \alpha^{\prime}-\delta .
$$

Fix $\bar{y} \in \Xi$ and let $\hat{y}=P_{s} \bar{y}, \tilde{y}=P_{u} \bar{y}, \hat{r}=|\hat{y}|$ and $\tilde{r}=|\tilde{y}|$. By (47) and the definition of $\gamma$ such $\delta$ exists. We have

$$
\begin{aligned}
(B \bar{y}, \tilde{y})+\left(M^{-1} N(M \bar{y}), \tilde{y}\right) & =(B \tilde{y}, \tilde{y})+\left(N(M \bar{y}),\left(M^{-1}\right)^{t} \tilde{y}\right) \\
& \geq \alpha^{\prime} \tilde{r}^{2}-\beta m_{1}^{2} m_{2}\left(\hat{r}^{2}+\tilde{r}^{2}\right) \tilde{r} \geq \delta \tilde{r}^{2},
\end{aligned}
$$

because $\hat{r} \leq \frac{k}{\gamma} \tilde{r}^{2}$ by the definition of $\Xi$. 
Lemma 10. Let $r, \alpha, \alpha^{\prime}, k, \hat{y}$ and $\kappa$ be as above. The unstable manifold tangent to $\hat{y}$ intersects $\kappa$.

Proof. By Lemma 9 the unstable manifold cannot be entirely contained in $\Xi$. By Lemmas 8 and 9 it can only exit through $\kappa$.

In the next subsection we apply these ideas in order to prove Theorem 4.

\subsection{The computer assisted proofs}

We apply the general result stated in the previous subsection to the system (8).

We first consider the point $P_{1}=(4,0,0,0)$. Let $x=v-P_{1}$. The system (8) takes the form (39) with

$$
A_{1}=\left[\begin{array}{cccc}
2-n & -1 & 0 & 0 \\
0 & 2 & 1 & 0 \\
0 & 0 & 4-n & 1 \\
0 & 0 & 0 & 4
\end{array}\right]
$$

$N(x)=\left(0,0,0, x_{1} x_{4}\right)$ and $\alpha=4-n$.

If we consider the linearization at $P_{2}=(0,4 n-8,16-8 n,-8(n-2)(n-4))$ and set $x=v-P_{2}$, then system (8) can be written as (39) with

$$
A_{2}=\left[\begin{array}{cccc}
2-n & -1 & 0 & 0 \\
0 & 2 & 1 & 0 \\
0 & 0 & 4-n & 1 \\
-8(-4+n)(-2+n) & 0 & 0 & 0
\end{array}\right]
$$

and again $N(x)=\left(0,0,0, x_{1} x_{4}\right)$. From Section 3.1 we know that if $n=5, \ldots, 12$ the eigenvalues are $\left((4-n) / 2+i \sigma,(4-n) / 2-i \sigma, \lambda_{1}, \lambda_{2}\right)$, where $\lambda_{1}<(4-n) / 2<0$ and $\lambda_{2}>0$. It turns out that $\alpha=(4-n) / 2$. If $n \geq 13$ all eigenvalues are real and

$$
\alpha=2-\frac{1}{2} n+\frac{1}{2} \sqrt{8-4 n+n^{2}-4 \sqrt{68-52 n+9 n^{2}}} .
$$

We remark that, since the nonlinear part is very simple, it is possible to obtain a better estimate for the coefficients $\beta, m_{1}$, and $m_{2}$ than the one we had in Section 6.1.

In the following, let $M$ be the matrix that diagonalizes either $A_{1}$ or $A_{2}$ and let $\left|M_{i}\right|$ be the (Euclidean) norm of the $i$-th row of $M$.

Lemma 11. For all $y_{1}, y_{2} \in \mathbb{R}^{4}$ the following inequality holds:

$$
\left(N\left(M y_{1}\right),\left(M^{-1}\right)^{t} y_{2}\right) \leq\left|M_{1}\right|\left|M_{4}\right|\left|\left(M^{-1}\right)_{4}^{t}\right|\left|y_{1}\right|^{2}\left|y_{2}\right|
$$

Proof. We have

$$
\left(N\left(M y_{1}\right),\left(M^{-1}\right)^{t} y_{2}\right)=\left(M y_{1}\right)_{1}\left(M y_{1}\right)_{4}\left(\left(M^{-1}\right)^{t} y_{2}\right)_{4}
$$


where we denoted by $(A v)_{i}$ the $i$-th component of the vector $(A v)$, i.e. the scalar product of the $i$-th row of $A$ with the vector $v$. The conclusion follows by the definition of $\left|M_{i}\right|$.

By the above lemma we infer that $\gamma$ may be obtained as

$$
\gamma=-\frac{\alpha}{\left|M_{1}\right|\left|M_{4}\right|\left|\left(M^{-1}\right)_{4}^{t}\right|}
$$

and $\varepsilon$ may be chosen arbitrarily.

To compute a rigorous enclosure $\left[\lambda_{\sigma}^{\min }, \lambda_{\sigma}^{\max }\right]$ for the value of $\lambda_{\sigma}$ we fix $n$ and we compute the value $\gamma$ in (48). We can choose $k>1$ and $r>0$ satisfying (44) and (47). We have some degree of arbitrariness: we prefer a small $r$ in order to have a small set $\kappa$, but we also like a large $r$ in order to reach the hyperplane with fewer time steps. It is also convenient to have the smallest possible $k$, since it also implies a smaller set $\kappa$. We have to make an empirical choice by trying different values and selecting the best trade-off. It turns out that it is convenient to choose $r$ first, set

$$
k=\frac{\gamma^{2}-\sqrt{\gamma^{4}-4 r^{2} \gamma^{2}}}{2 r^{2}}
$$

and check whether (47) holds. Since the unstable manifold in $P_{2}$ is one-dimensional, we have to choose between two possible directions. The numerical experiment gave us the correct direction. Once we choose $r$ and compute $k$ we have the set $\kappa$ as given in (43). We should compute the evolution of all points in $\kappa$ and its intersection with the hyperplane $v_{1}=4$. This would require a very long computer time, but since two solutions of (39) cannot intersect, then it is enough to compute the evolution of the points in the boundary of $\kappa$, provided we can prove that the trajectories of all point in the interior of $\kappa$ also reach the hyperplane $v_{1}=4$. This can be checked by the following lemma.

Lemma 12. Set

$$
\kappa^{\prime}:=\kappa_{\hat{y}}^{\prime}:=\hat{y}+\left\{\tilde{y} \in S_{0}:|\tilde{y}|=\frac{k}{\gamma} r^{2}\right\} .
$$

Assume that the trajectories of all points in $\kappa^{\prime}$ intersect the hyperplane $v_{1}=4$ and do not intersect the hyperplane $(2-n) v_{1}-v_{2}+4(n-2)=0$. Let $\hat{\kappa}$ be the intersection of all such trajectories with $v_{1}=4$.

Then the trajectories of all points in $\kappa$ also intersect the hyperplane $v_{1}=4$ and the intersection takes place in the region bounded by $\hat{\kappa}$.

Proof. Since $v_{1}^{\prime}=(2-n) v_{1}-v_{2}+4(n-2)$, then $v_{1}^{\prime}$ is positive and bounded away from zero for all points of the trajectories starting from $\kappa^{\prime}$. Then, by the uniqueness and continuous dependence on the initial condition of the Cauchy problem, it follows that the union $\tau\left(\kappa^{\prime}\right)$ of such trajectories is a "tube" in $\mathbb{R}^{4}$ and the trajectories of all points in $\kappa \backslash \kappa^{\prime}$ cannot exit $\tau\left(\kappa^{\prime}\right)$. Then $v_{1}^{\prime}$ is also positive and bounded away from zero for all points starting in $\kappa$ and the trajectory of every point in $\kappa$ reaches $v_{1}=4$ in a finite time.

Our strategy is as follows: we compute the intersection of the flow starting from all points in $\kappa^{\prime}$ with the hyperplane $v_{1}=4$. If all the trajectories intersect the hyperplane, we have a proof that the singular solution exists; furthermore the envelope in the $v_{4}$-direction of all intersections yields the desired $\lambda$-interval. Note that the set $\kappa^{\prime}$ is the image of $S^{2}$ through an invertible affine map, therefore we need an efficient discretization of a sphere. 
Lemma 13. For all $n=5, \ldots, 16$ let $r=.001$, let $k$ be as in (49) and $\kappa^{\prime}=\kappa_{\hat{y}}^{\prime}$ as in (50). For a suitable choice of the direction $\hat{y}$ in the one dimensional unstable manifold $U_{0}$, the following conclusions hold:

1. The flow starting in $\kappa^{\prime}$ intersects the hyperplane $v_{1}=4$.

2. The absolute value of the first coordinate of the intersection point is in the interval set $\left[\lambda_{\sigma}^{\min }, \lambda_{\sigma}^{\max }\right]$ defined in $(10)$.

3. The flow starting in $\kappa^{\prime}$ and ending on the hyperplane $v_{1}=4$ does not intersect the hyperplane $(2-n) v_{1}-v_{2}+4(n-2)=0$.

The proof is by computer assistance, as described in Section 9.

In order to compute a rigorous lower bound for $\lambda^{*}$ we consider the trajectories of points in the unstable manifold of $P_{1}$ and compute the intersection with the hyperplane $v_{1}=4$. Since the manifold is two dimensional, we have to decide the direction to follow: we use the numerical results presented above to compute the direction that gives the highest possible value for $\lambda$. We define $\kappa$ as above, and we wish to prove that all trajectories starting from $\kappa$ intersect the hyperplane $v_{1}=4$. We also need to estimate the location of such intersections. It would save some computer time to restrict the computation to the boundary of $\kappa$ as in the proof of Lemma 13, but we cannot proceed as in Lemma 12 because $P_{1}$ lies on the hyperplane $v_{1}=4$ and therefore $v_{1}$ cannot be monotone. Furthermore, since the unstable manifold has now dimension 2, we do not have the topological argument (the tube) used before. On the other hand, in this case we only have to consider a region which is the affine image of a disk, therefore it is feasible to compute the trajectory for all point in the disk.

Lemma 14. For all $n=5, \ldots, 10$, let $r=.001$ if $n \leq 9$ and $r=.0001$ if $n=10$; let $\hat{y}=P_{1}+$ $r\left(e_{1} \sin \vartheta_{n}+e_{2} \cos \vartheta_{n}\right)$, where $e_{1}$ and $e_{2}$ are the eigenvectors of $A_{1}$ with unit norm and positive first component corresponding respectively to the eigenvalues 2 and 4 and $\vartheta_{5}=6.2829856$, $\vartheta_{6}=6.28298854, \vartheta_{7}=6.2829901, \vartheta_{8}=6.2829918, \vartheta_{9}=6.2829914, \vartheta_{10}=6.28316589$, let $k$ be as in (49) and let $\kappa$ be as in (43).

1. The flow starting at all points of $\kappa$ intersects the hyperplane $v_{1}=4$.

2. The absolute value of the first coordinate of the intersection point is larger than $\lambda_{\min }^{*}$ displayed in (10).

We point out that this statement only shows that there exists a regular solution for some value of $\lambda$ obtained as the intersection of a one-dimensional submanifold of the unstable manifold with the hyperplane $v_{1}=4$. Since we cannot exclude that there exists a solution for a larger value of $\lambda$, we only have a lower bound for $\lambda^{*}$.

The proof of Theorem 4 follows by Lemmas 10-14.

\section{Proof of Theorem 5}

In this section we use both the PDE notation $\Delta^{2}$ and the ODE notation with primes denoting differentiation (with respect to $r$ or $s$, depending on the context). 
We assume that $U_{\sigma}$ is any radial weakly singular solution of $\left(P_{\lambda_{\sigma}}\right)$ with

$$
\lambda_{\sigma}>8(n-2)(n-4)
$$

In particular, we deal with those solutions obtained in Theorem 4, see also Table (10). Then, by Theorem 3 (ii), we know that

$$
U_{\sigma}(r)=-4 \log r+o(|\log r|) \quad \text { as } r \rightarrow 0 .
$$

Therefore, we define the function

$$
W(r):=U_{\sigma}(r)+4 \log r
$$

and we study its behaviour. After some calculations, we find that it weakly solves the equation

$$
\begin{cases}\Delta^{2} W=\frac{1}{|x|^{4}}\left[\lambda_{\sigma} e^{W}-8(n-2)(n-4)\right] & \text { in } B \\ W=0 & \text { on } \partial B \\ \frac{\partial W}{\partial \mathbf{n}}=4 & \text { on } \partial B .\end{cases}
$$

The proof of Theorem 5 follows from the next two lemmas and Proposition 1 at the end of this section.

Lemma 15. Assume (51) and assume that $W \in C^{4}(0,1]$ weakly solves $(52)(W=W(r))$; then

$$
\lim _{r \rightarrow 0} W(r)=\log \frac{8(n-2)(n-4)}{\lambda_{\sigma}}=W_{0}<0 .
$$

Moreover, at least one of the two following facts holds true:

(i) the function $W(r)-W_{0}$ changes sign infinitely many times in any neighbourhood of $r=0$;

(ii) $W(r) \geq \max \left[W_{0}, 2 r^{2}-2\right]$ for all $r \in(0,1]$.

Proof. The negativity of $W_{0}$ follows from (51) while (53) is a consequence of Theorem 3.

Assume that case $(i)$ in the statement does not occur; we first claim that

$$
W(r) \geq W_{0} \quad \text { for all } r \in(0,1] .
$$

For contradiction, assume that (54) does not hold; then, there exists $\bar{R} \in(0,1)$ such that $W(\bar{R})<W_{0}$ and two cases may occur:

First case: there exists $R \in(0,1)$ such that $W^{\prime}(R)=0$ and $W_{0} \leq W(r)<W(R)$ for all $r \in(0, R)$. In this case, let $H(r)=W(r)-W(R)$ so that $H(r)<0$ for all $r \in(0, R)$; on the other hand, $H$ weakly solves the problem

$$
\begin{cases}\Delta^{2} H=\Delta^{2} W \geq 0 & \text { in } B_{R} \\ H=\frac{\partial H}{\partial \mathbf{n}}=0 & \text { on } \partial B_{R}\end{cases}
$$

so that by Lemma 1 , one gets $H(r) \geq 0$ for all $r \in(0, R)$, a contradiction. 
Second case: there exists $R \in(0,1)$ such that $W^{\prime}(R)=0$ and $W_{0} \geq W(r)>W(R)$ for all $r \in(0, R)$. In this case, $H(r)=W(r)-W(R)$ satisfies both $H(r)>0$ for all $r \in(0, R)$ and

$$
\begin{cases}\Delta^{2} H=\Delta^{2} W \leq 0 & \text { in } B_{R} \\ H=\frac{\partial H}{\partial \mathbf{n}}=0 & \text { on } \partial B_{R}\end{cases}
$$

giving again a contradiction.

We have so proved (54): hence, if we define the function $\phi(r)=W(r)+2-2 r^{2}$ we infer that $\phi=\phi(|x|)$ weakly satisfies

$$
\begin{cases}\Delta^{2} \phi=\Delta^{2} W \geq 0 & \text { in } B \\ \phi=\frac{\partial \phi}{\partial \mathbf{n}}=0 & \text { on } \partial B ;\end{cases}
$$

this yields $\phi(r) \geq 0$, namely $W(r) \geq 2 r^{2}-2$ for all $r \in(0,1]$.

We have so proved that if $(i)$ does not occur then $(i i)$ holds true, that is, the statement.

In high dimensions the previous alternative breaks down and we can describe the behaviour of weakly singular solutions:

Lemma 16. If $n \geq 13$, then case ( $i$ ) of Lemma 15 cannot occur.

Proof. Let $W=W(r)$ and $W_{0}$ be as in Lemma 15 and consider the function

$$
Z(s)=W\left(e^{s}\right)-W_{0} \quad s \in(-\infty, 0) .
$$

Then, since $W$ satisfies (52), we deduce that

$$
L_{4} Z+p(s) Z=0 \quad s \in(-\infty, 0),
$$

where $L_{4} Z=Z^{\prime \prime \prime \prime}+2(n-4) Z^{\prime \prime \prime}+\left(n^{2}-10 n+20\right) Z^{\prime \prime}-2(n-2)(n-4) Z^{\prime}$ and

$$
p(s)=-8(n-2)(n-4) \frac{e^{Z(s)}-1}{Z(s)} .
$$

Note that $p(s)$ is well-defined for all $s<0$ and that, by (53), $p(s) \rightarrow-8(n-2)(n-4)$ as $s \rightarrow-\infty$. In particular, for all $\varepsilon>0$ there exists $s_{\varepsilon}<0$ such that

$$
p(s) \geq-[8(n-2)(n-4)+\varepsilon] \text { for all } s \leq s_{\varepsilon} .
$$

Since $n \geq 13$, for sufficiently small $\varepsilon$, the linear equation

$$
L_{4} Z-[8(n-2)(n-4)+\varepsilon] Z=0
$$

admits four linearly independent solutions of "exponential type", namely $Z_{i}(s)=e^{\nu_{i} s}$ for some $\nu_{i} \in \mathbb{R}(i=1, \ldots, 4)$, see also the discussion in Section 3.1. Hence, (57) is nonoscillatory in $(-\infty, 0)$ according to the definition in [E]. Therefore, by (56) and [E, Corollary 1], also $(55)$ is nonoscillatory in $(-\infty, 0)$ and the statement follows.

Let us conclude this section with the observation that an explicit form of the weakly singular solution $U_{\sigma}$ seems not so easy to be obtained: 
Proposition 1. Assume that the function $W$ is a solution of (52) as considered in Lemma 15. Then the function $W=W(r)$ is not analytic in $r$ close to 0 .

Proof. For contradiction, let $a_{k}=W^{(2 k)}(0) /(2 k)$ ! and assume that

$$
W(r)=\sum_{k=0}^{\infty} a_{k} r^{2 k}
$$

is a convergent power series for $r$ close to 0 . Since $W$ is regular, the r.h.s. of the equation in (52) is bounded as $r \rightarrow 0$ and we necessarily have

$$
a_{0}=\log \frac{8(n-2)(n-4)}{\lambda_{\sigma}}, \quad a_{1}=\frac{W^{\prime \prime}(0)}{2}=0 .
$$

Then,

$$
W^{(k)}(r)=\frac{W^{\prime \prime \prime \prime}(0)}{(4-k) !} r^{4-k}+O\left(r^{5-k}\right) \quad \text { as } r \rightarrow 0 \quad(k=1,2,3)
$$

and hence

$$
\frac{n(n+2)}{3} W^{\prime \prime \prime \prime}(0)=\left.\Delta^{2} W\right|_{r=0}=\frac{\lambda_{\sigma} e^{a_{0}} W^{\prime \prime \prime \prime}(0)}{24}=\frac{(n-2)(n-4)}{3} W^{\prime \prime \prime \prime}(0)
$$

where we have used (52) and (58). This shows that $W^{\prime \prime \prime \prime}(0)=0$ and $a_{2}=0$.

We now proceed by induction. Assume that for some $k \geq 2$ we have shown that $a_{1}=\ldots=$ $a_{k}=0$; we claim that $a_{k+1}=0$. Once we show this, we achieve a contradiction and the statement follows. Note that $\lambda_{\sigma} e^{W}-8(n-2)(n-4)=8(n-2)(n-4)\left[e^{W-a_{0}}-1\right]$ and, by induction assumption,

$$
\frac{1}{r^{4}}\left(e^{W-a_{0}}-1\right)=a_{k+1} r^{2 k-2}+O\left(r^{2 k}\right) .
$$

Therefore, from (52) we get:

$$
\left.\left(\frac{d}{d r}\right)^{2 k-2} \Delta^{2} W\right|_{r=0}=8(2 k-2) !(n-2)(n-4) a_{k+1} .
$$

On the other hand, recalling the radial form of $\Delta^{2}$ (see the l.h.s. of (4)) and taking into account that $($ as $r \rightarrow 0)$

$$
\begin{aligned}
W^{\prime}(r) & \sim \frac{W^{(2 k+2)}(0)}{(2 k+1) !} r^{2 k+1}, & W^{\prime \prime}(r) & \sim \frac{W^{(2 k+2)}(0)}{(2 k) !} r^{2 k}, \\
W^{\prime \prime \prime}(r) & \sim \frac{W^{(2 k+2)}(0)}{(2 k-1) !} r^{2 k-1}, & W^{\prime \prime \prime \prime}(r) & \sim \frac{W^{2 k+2}(0)}{(2 k-2) !} r^{2 k-2} .
\end{aligned}
$$

We also deduce that

$$
\left.\left(\frac{d}{d r}\right)^{2 k-2} \Delta^{2} W\right|_{r=0}=2 k(2 k+2)(n+2 k)(n+2 k-2) \cdot(2 k-2) ! a_{k+1} .
$$

Combining this with (59), we get

$$
a_{k+1}\{2 k(2 k+2)(n+2 k)(n+2 k-2)-8(n-2)(n-4)\}=0 .
$$

Since the term in brackets is strictly positive, this yields $a_{k+1}=0$. 


\section{Further results and open problems}

First, we discuss the stability of the linearizations around regular solutions of problem $\left(P_{\lambda}\right)$. For this purpose we observe that the minimal solution depends continuously on $\lambda$ :

Proposition 2. Let as before denote $U_{\lambda}$ the minimal solution of $\left(P_{\lambda}\right)$. Then, $\left[0, \lambda^{*}\right) \ni \lambda \mapsto$ $U_{\lambda} \in C^{4, \alpha}(\bar{B})$ is continuous from the left. Moreover, if $\lambda_{0} \in\left[0, \lambda^{*}\right)$ is such that the first eigenvalue of the linearization $L_{U_{\lambda_{0}}}:=\Delta^{2}-\lambda_{0} \exp \left(U_{\lambda_{0}}\right)$ is strictly positive, then $\lambda \mapsto U_{\lambda}$ is also continuous in $\lambda=\lambda_{0}$.

Proof. Let $\lambda_{k} \nearrow \lambda_{0}$. Since $U_{\lambda_{k}} \leq U_{\lambda_{0}}$ and since the $\left(U_{\lambda_{k}}\right)_{k}$ are monotonically increasing, we get $\tilde{U}:=\lim _{k \rightarrow \infty} U_{\lambda_{k}}$ first in any $L^{q}$-space, then by elliptic theory in $W^{4, q}$ and finally in $C^{4, \alpha}(\bar{B})$. Hence, also $\tilde{U}$ solves $\left(P_{\lambda_{0}}\right)$, and $0<\tilde{U} \leq U_{\lambda_{0}}$. We conclude that $\tilde{U}=U_{\lambda_{0}}$ by minimality of $U_{\lambda_{0}}$.

The second statement follows from the implicit function theorem and again the monotonicity of $U_{\lambda}$ in $\lambda$.

The next statement extends some results of [CR] to the biharmonic case, see Proposition 2.15 there. In order to show the sign condition of eigenfunctions we use a decomposition method with respect to pairs of dual cones.

Proposition 3. Let $u$ be a regular solution for $\left(P_{\lambda}\right)$, where $\lambda \in\left(0, \lambda^{*}\right]$. Let the first eigenvalue $\mu_{1}$ of the linearization $L_{u}:=\Delta^{2}-\lambda e^{u}$ under Dirichlet boundary conditions be nonnegative: $\mu_{1} \geq 0$. Then every eigenfunction of $L_{u} \varphi=\mu_{1} \varphi$ is of fixed sign. Moreover, if $v \in C^{4}(\bar{B})$ solves $\Delta^{2} v \geq \lambda e^{v}$ in $B$ and $v=\frac{\partial v}{\partial \mathbf{n}}=0$ on $\partial B$, then it follows that $v \geq u$. Finally, if $\mu_{1}=0$, then we even have $v=u$.

Proof. In order to show that the first eigenfunction $\varphi$ of $L_{u}$ is of fixed sign, we need to explain a decomposition technique with respect to dual cones, which was found in the abstract setting by Moreau [Mo] and adapted to biharmonic Dirichlet problems in [GG]. As usual we equip $H_{0}^{2}(B)$ with the scalar product

$$
(u, w)_{H_{0}^{2}}:=\int_{B} \Delta u \Delta w d x
$$

Let here denote

$$
\mathcal{K}=\left\{u \in H_{0}^{2}(B) ; u \geq 0 \text { a.e. in } B\right\},
$$

the convex closed cone of nonnegative $H_{0}^{2}$-functions and

$$
\mathcal{K}^{\prime}=\left\{u \in H_{0}^{2}(B) ; \quad \text { for all } w \in \mathcal{K}: \quad(u, w)_{H_{0}^{2}} \leq 0\right\}
$$

its dual cone in $H_{0}^{2}$ of weak subsolutions of the clamped plate equation. By Lemma 1 we see that $\mathcal{K}^{\prime} \subset-\mathcal{K}$. For any $w \in \mathcal{K}^{\prime}$ we even have that either $w \equiv 0$ or $w<0$ in $B$.

Assume now by contradiction that $\varphi$ is not of fixed sign. Then, according to [Mo], we may decompose

$$
\varphi=\varphi_{1}+\varphi_{2}
$$


with $\varphi_{1} \in \mathcal{K}, \varphi_{2} \in \mathcal{K}^{\prime}$ and $\varphi_{1} \perp \varphi_{2}$ in $H_{0}^{2}(B)$. By assumption we have that $\varphi_{1} \geq 0, \varphi_{1} \not \equiv 0$ and $\varphi_{2}<0$. But then

$$
\begin{aligned}
0 \leq \mu_{1} & =\inf _{w \in H_{0}^{2}(B) \backslash\{0\}} \frac{\int_{B}\left((\Delta w)^{2}-\lambda \exp (u) w^{2}\right) d x}{\int_{B} w^{2} d x} \\
& \leq \frac{\int_{B}\left(\left(\Delta\left(\varphi_{1}-\varphi_{2}\right)\right)^{2}-\lambda \exp (u)\left(\varphi_{1}-\varphi_{2}\right)^{2}\right) d x}{\int_{B}\left(\varphi_{1}-\varphi_{2}\right)^{2} d x} \\
& <\frac{\int_{B}\left(\left(\Delta\left(\varphi_{1}+\varphi_{2}\right)\right)^{2}-\lambda \exp (u)\left(\varphi_{1}+\varphi_{2}\right)^{2}\right) d x}{\int_{B}\left(\varphi_{1}+\varphi_{2}\right)^{2} d x} \\
& =\frac{\int_{B}\left((\Delta \varphi)^{2}-\lambda \exp (u) \varphi^{2}\right) d x}{\int_{B} \varphi^{2} d x}=\mu_{1},
\end{aligned}
$$

a contradiction. Hence, $\varphi$ is of fixed sign, say $\varphi \geq 0$, and in a second step we may conclude from the equation and the strict positivity of the biharmonic Green function (in the ball) that $\varphi>0$.

We consider now $u$ and $v$ as in the statement. For $\tau \in[0,1]$ we look at

$$
\begin{aligned}
& \Delta^{2}(u+\tau(v-u))-\lambda \exp (u+\tau(v-u)) \\
& \quad \geq \Delta^{2}(u+\tau(v-u))-\lambda(\tau \exp (v)+(1-\tau) \exp (u)) \geq 0 .
\end{aligned}
$$

Since (60) equals 0 for $\tau=0$, its first derivative at $\tau=0$ must be nonnegative:

$$
\Delta^{2}(v-u)-\lambda e^{u}(v-u)=: f \geq 0
$$

If $\mu_{1}>0$ a decomposition trick as above applied to the functional $w \mapsto \int_{B}\left((\Delta w)^{2}-\lambda e^{u} w^{2}-\right.$ $f w) d x$ shows that $v \geq u$.

If $\mu_{1}=0$, we test (61) with the positive first eigenfunction $\varphi$ and get

$$
\Delta^{2}(v-u)-\lambda e^{u}(v-u)=0
$$

That means that also the first derivative of (60) with respect to $\tau=0$ vanishes, so that the second derivative needs to be nonnegative:

$$
-\lambda e^{u}(v-u)^{2} \geq 0
$$

But this immediately yields $v=u$.

Concerning the stability behaviour of the linearizations around regular solutions, we have:

Proposition 4. Let $\lambda>0$, let $u$ be a regular solution of $\left(P_{\lambda}\right)$, let $L_{u}=\Delta^{2}-\lambda e^{u}$ be the linearized operator at $u$ and let $\mu_{1}=\mu_{1}\left(L_{u}\right)$ be the smallest eigenvalue of $L_{u}$; then

(i) if $\lambda<\lambda^{*}$ and $u$ is the minimal solution, then $\mu_{1}>0$;

(ii) if $\lambda<\lambda^{*}$ and $u$ is not the minimal solution, then $\mu_{1}<0$;

(iii) if $\lambda=\lambda^{*}$ and the extremal solution $u=U_{*}$ is regular, then $\mu_{1}=0$.

Finally, if $U_{\lambda}$ denotes the minimal (regular) solution of $\left(P_{\lambda}\right)$ and $\mu_{1}(\lambda)=\mu_{1}\left(L_{U_{\lambda}}\right)$, then, the map $\lambda \mapsto \mu_{1}(\lambda)$ is decreasing. 
Proof. (i) The monotonicity of $\mu_{1}(\lambda)$ follows immediately from the variational characterization

$$
\mu_{1}(\lambda)=\inf _{w \in H_{0}^{2}(B) \backslash\{0\}} \frac{\int_{B}(\Delta w)^{2} d x-\int_{B} \exp \left(U_{\lambda}\right) w^{2} d x}{\int_{B} w^{2} d x}
$$

and from the monotonicity of $U_{\lambda}$ with respect to $\lambda$. By Proposition 2 we see that the function $\lambda \mapsto \mu_{1}(\lambda)$ is continuous from the left on $\left(0, \lambda^{*}\right)$ and even on $\left(0, \lambda^{*}\right]$, provided the extremal solution $U_{*}$ is regular.

Assume by contradiction that there exists a $\tilde{\lambda} \in\left(0, \lambda^{*}\right)$ with $\mu_{1}(\tilde{\lambda}) \leq 0$. We put

$$
\lambda_{0}:=\sup \left\{\lambda \geq 0: \mu_{1}(\lambda)>0\right\} \leq \tilde{\lambda}<\lambda^{*} .
$$

According to the mentioned continuity from the left, we have $\mu_{1}\left(\lambda_{0}\right) \geq 0$. If we assume $\mu_{1}\left(\lambda_{0}\right)>0$, then the second part of Proposition 2 would give $\mu_{1}(\lambda)>0$ also for some $\lambda>\lambda_{0}$, a contradiction. Consequently we have $\mu_{1}\left(\lambda_{0}\right)=0$. Let $u=U_{\lambda_{0}}>0$ be the corresponding minimal solution:

$$
\Delta^{2} u=\lambda_{0} e^{u} \text { in } B, \quad u=\nabla u=0 \text { on } \partial B .
$$

Consider any $\lambda \in\left(\lambda_{0}, \lambda^{*}\right)$ with minimal solution $v=U_{\lambda}>0$ :

$$
\Delta^{2} v=\lambda e^{v} \text { in } B, \quad v=\nabla v=0 \text { on } \partial B
$$

Since $\lambda>\lambda_{0}$, Proposition 3 applies and yields $v=u$ and hence $\lambda=\lambda_{0}$, a contradiction.

(ii) Let $U_{\lambda}$ be the minimal solution for $\left(P_{\lambda}\right)$ so that $u \geq U_{\lambda}$. If the linearization around $u$ had nonnegative first eigenvalue, then Proposition 3 would also yield $u \leq U_{\lambda}$ so that $u$ and $U_{\lambda}$ necessarily coincide, contradiction.

(iii) Assume that the extremal solution $u=U_{*}$ is regular. By continuity, we have $\mu_{1} \geq 0$. If $\mu_{1}>0$ the implicit function theorem would also yield solutions for some $\lambda>\lambda^{*}$. A contradiction, so that $\mu_{1}=0$.

Open Problem 1. Does $(i i)$ of Proposition 4 extend to weak solutions $u$ as formulated in $[\mathrm{BV}$, Theorem 3.1]?

We now turn to the extremal solution $U_{*}$. We first suggest the

Open Problem 2. Do we have uniqueness of weak solutions for $\left(P_{\lambda^{*}}\right)$ ? By Proposition 4 (iii) and arguing as in Lemma 2.6 in [BV] one obtains that if the extremal solution is regular then it is unique even in a weak sense. However, without the regularity assumption on $U_{*}$, the proof seems much more difficult; we refer to [Ma] for the corresponding result related to the second order problem (1). In particular, the proof of a result in the spirit of [Ma, Lemma 2.1] requires a new trick, probably of the same kind as the one we used to prove Lemma 5.

Perhaps, the precise characterization of all singular solutions $U_{\sigma}$ and the corresponding "singular" parameters $\lambda_{\sigma}$ is the most interesting and difficult problem we have to leave open in the present paper.

Open Problem 3. Is the singular parameter and the weakly singular solution unique? In order to construct a weakly singular radial solution, according to Theorem 3, one has to follow the unstable branch arising from $P_{2}$. One can do so in two (opposite) exit directions. In one direction we actually find at most (and presumably precisely) one solution by the 
result of Soranzo [So]: the solution of the PDE has to be strictly decreasing. We emphasize that this result extends to the class of weakly singular radial solutions. For the ODE system (8) this means that any "singular" trajectory may intersect the hyperplane $v_{1}=4$ only once and cannot come back to it. But we do not have a proof that the unstable branch leaving $P_{2}$ in the other direction will not intersect the hyperplane $v_{1}=4$ even if numerical experiments suggest so.

Next, we recall that in [GGM] it was shown that for any open bounded domain $\Omega \subset \mathbb{R}^{n}$ there exist $C_{1}, C_{2}>0$ such that the following improved Hardy's inequality holds:

$$
\int_{\Omega}|\Delta u|^{2} d x \geq \frac{n^{2}(n-4)^{2}}{16} \int_{\Omega} \frac{u^{2}}{|x|^{4}} d x+C_{1} \int_{\Omega} \frac{u^{2}}{|x|^{2}} d x+C_{2} \int_{\Omega} u^{2} d x \quad \text { for all } u \in H_{0}^{2}(\Omega) .
$$

A similar inequality was used in $[\mathrm{BV}]$ in order to establish the space dimensions in which the extremal solution for $(1)$ is regular or singular. For $\left(P_{\lambda}\right)$ this seems more intriguing: it is not clear which is the role of the two remainder terms in (62). Furthermore, as we have seen in Theorem 5 and Proposition 1, the singular solution is difficult to describe. However, we have a partial result relating Hardy's inequality with extremal solutions: clearly, this statement is weaker than Corollary 1 if $n \leq 10$.

Proposition 5. Let $\lambda_{\sigma}$ and $U_{\sigma}$ be as in Theorem 4 and assume that $\lambda_{\sigma}=\lambda^{*}$. Then, if $n \leq 12$, case (ii) in Theorem 5 cannot occur.

Proof. By Proposition $4($ i), by Theorem 1 (ii)-(iii) and using the notations of Theorem 5 , we infer that

$$
\int_{B}|\Delta \phi|^{2} \geq \lambda^{*} \int_{B} e^{U_{*}} \phi^{2}=\lambda^{*} \int_{B} \frac{e^{W}}{|x|^{4}} \phi^{2} \quad \text { for all } \phi \in H_{0}^{2}(B) .
$$

For contradiction, if $(i i)$ in Theorem 5 holds, then

$$
\lambda^{*} \int_{B} \frac{e^{W}}{|x|^{4}} \phi^{2} \geq 8(n-2)(n-4) \int_{B} \frac{\phi^{2}}{|x|^{4}} \quad \text { for all } \phi \in H_{0}^{2}(B) .
$$

Since $8(n-2)(n-4)>\frac{n^{2}(n-4)^{2}}{16}$ whenever $n \leq 12$, the last inequality, together with (63), would improve the best constant in Hardy's inequality, a contradiction.

Proposition 5 and Corollary 1 suggest the following question and conjecture:

Open Problem 4. Which are all the space dimensions $n \geq 5$ for which $\lambda_{\sigma}<\lambda^{*}$ ? We conjecture that the answer is $n \leq 12$. In view of Corollary 1 we know that among these dimensions $n$ there are at least $5 \leq n \leq 10$. Moreover, Theorem 5 and Proposition 5 prove "half" of this conjecture when $n=11,12$. Maybe the proof relies on the interpretation of the two remainder terms in (62).

Open Problem 5. Show that any radial singular solution is also weakly singular, according to Definition 3. In particular, this would strengthen the statement of Theorem 3.

If the previous three open problems could be solved in the affirmative, then we could also conclude that the extremal solution $U_{*}$ is singular if and only if $n \geq 13$.

We conclude this paper with some further problems. The next one is not yet completely solved even in the second order case. 
Open Problem 6. Do there exist singular nonradial solutions to $\left(P_{\lambda}\right)$ for some $\lambda>0$ ? We conjecture that the answer is positive, see also Problem 7 in $[\mathrm{BV}]$.

The following picture displays the numerically computed value of $-v_{4}$ of the intersection of a portion of the unstable manifold of $P_{1}$ with the hyperplane $v_{1}=4$ in the case $n=5$.

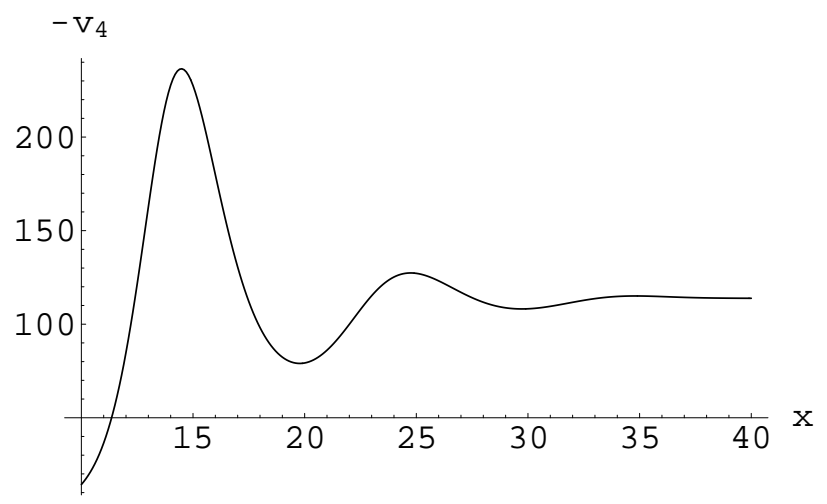

More precisely, $-v_{4}$ is displayed as a function of $x:=-\log (-\vartheta)$. One may observe the estimated value of $\lambda^{*}$ as the maximum value reached by $-v_{4}$; furthermore, as $\vartheta \rightarrow 0^{-}$the value of $-v_{4}$ appears to asymptotically reach $\lambda_{\sigma}$ oscillating around it. This leads us to the following

Open Problem 7. Assume $n \leq 12$. Prove that for every $N \in \mathbb{N}$ there exists $\varepsilon=\varepsilon(N)>0$ such that for $\lambda \in\left[\lambda_{\sigma}-\varepsilon, \lambda_{\sigma}+\varepsilon\right]$ there exist at least $N$ distinct regular radial solutions. For the second order problem the same statement holds true, see [GPP, Theorem 15].

Open Problem 8. How can one proceed in arbitrary smooth domains where it is known that comparison principles like Lemma 1 become false? How can one construct and characterize the minimal solution? Does one have similar bifurcation diagrams as here, while only the solutions do possibly have small regions, where they become negative? Or does the lack of comparison principles lead to a completely different behaviour, at least in geometrically very complicated domains?

\section{Appendix: computation techniques}

We describe here the algorithm used in the computer assisted proofs. In order to prove Theorem 4 we need a rigorous estimate of the intersection of a branch of the unstable manifold with the hyperplane $v_{1}=4$. Since we do not know the exact location of any point of the manifold, except for the stationary point, we compute the trajectory of the whole set $\kappa^{\prime}$ as described in Section 6. Since no analytical solution of the equation is available, we estimate the trajectories of all points of the set and compute the intersections with the hyperplane $v_{1}=4$ with rigorous error bounds. In order to compute the image of an infinite set of points, we partition it in boxes with small enough sides, which we call interval sets and we compute their trajectory using interval arithmetics. More precisely, we start with a Taylor approximation of order 10, i.e. we estimate the trajectory of an interval by using the Taylor expansion of order 10 and we estimate the error by the Lagrange remainder. If $h$ is the time step, we compute a rough but rigorous enclosure $D$ of the trajectory at times $[0, h]$, that is an interval set $D$ such that the solution of the equation lies in $D$ for all times between 0 and $h$. By 
Lagrange theorem we estimate the error we make neglecting the remaining terms of the Taylor expansion by computing $x^{(11)}(D) \frac{h^{11}}{11 !}$. We compute $x^{(11)}(D)$ (which is an interval enclosing all possible values assumed by the 11th derivative of the trajectory, therefore enclosing the Lagrange remainder) using a recursive algorithm for the time derivatives of the solutions (see Section I.8 in $[\mathrm{HNW}])$. We point out that it takes a finite amount of $s$-time to go from any point in $\kappa^{\prime}$ to the hyperplane $v_{1}=4$.

The interval arithmetics algorithms address the problem of computing the trajectory of an interval and of keeping track of the errors in an elegant and rigorous way, but they introduce another problem. Indeed, even in the simplest dynamical system, the procedure described above leads to a very rough estimate of trajectories, due to the wrapping effect which makes the bounds on the error grow exponentially fast. The wrapping effect is one of the main problem one faces when trying to do rigorous numerics for ordinary differential equations.

We describe it with one example: consider a square centered at the origin $x=[-\delta, \delta]^{2}$ and the matrix that represents the rotation in $\mathbb{R}^{2}$ by an angle $\alpha$

$$
R(\alpha)=\left[\begin{array}{cc}
\cos (\alpha) & -\sin (\alpha) \\
\sin (\alpha) & \cos (\alpha)
\end{array}\right]
$$

Assume for simplicity that $0<|\alpha|<\pi / 2$. If we apply $R_{\alpha}$ to $x$ and wish to represent the result by another interval (i.e. another rectangle with sides parallel to the coordinate axes), we see that we need $(\cos (\alpha)+|\sin (\alpha)|)[-\delta, \delta]^{2}$, therefore, although $R_{\alpha}$ is an isometry, its computer realization has a growth factor $\cos (\alpha)+|\sin (\alpha)|>1$. When solving the system of equations of the harmonic oscillator

$$
\dot{x}=-y, \quad \dot{y}=x
$$

the $2 \pi$-shift along the trajectory is an identity map, but when we compute it numerically in interval arithmetics, say with time step $h=2 \pi / N$, we have to compose $N$ times the map induced by $R(h)$. An easy computation shows this computation yields a growth factor $e^{2 \pi} \approx 535$ as $h \rightarrow 0$.

We substantially reduce the wrapping effect by using the Lohner algorithm. A complete description of interval arithmetics and of the Lohner algorithm is beyond the scope of this paper; we refer to Section 6 in [AZ] and references cited there for an exhaustive treatment of the topic. More specifically, see [MZ] concerning interval arithmetics and [Lo] for the Lohner algorithm. For the purpose of this description it suffices to consider the Lohner algorithm as a finite number of interval arithmetic operations based on the Taylor expansion which, given equation (8), an (interval) initial condition $V_{0} \subset \mathbb{R}^{4}$ and a time step $h$, returns an interval $V_{1} \subset \mathbb{R}^{4}$ such that for all points $v_{0} \in V_{0}$ the solution $v(s)$ of the Cauchy problem with initial condition $v(0)=v_{0}$ satisfies $v(h) \in V_{1}$. In other words, the Lohner algorithm provides a rigorous enclosure of the solution at time $h$ of a given Cauchy problem by performing a finite number of operations. The fact that the operations involved are finite and purely arithmetical makes it suitable for the implementation with a computer.

We have to determine the intersection of the trajectory with the hyperplane $v_{1}=4$. Since we are computing the trajectory of an interval, it takes a finite (nonzero) amount of 'time' to cross the hyperplane, therefore we necessarily introduce another error when estimating the intersection point and we have to give a rigorous bound for this error as well. We proceed as 
follows. We numerically compute the time $s_{1}$ required for the flow to reach the intersection. We compute with the Lohner algorithm the solution $V_{1}$ of the problem at time $s_{1}$. We check if the first component $\left(V_{1}\right)_{1}$ of $V_{1}$ is contained in $(-\infty, 4]$. If $\left(V_{1}\right)_{1} \subset(-\infty, 4]$, then no points in $V_{1}$ have crossed the hyperplane. If $\left(V_{1}\right)_{1} \not \subset(-\infty, 4]$ we choose (arbitrarily) a smaller value of $s_{1}$ and repeat the step. Then we roughly compute the time $s_{2}$ required for the set $V_{1}$ to cross the hyperplane. With the Lohner algorithm we compute the solution $V_{2}$ of the problem at time $s_{2}$. We check that all points in $V_{2}$ have crossed the hyperplane, i.e. $\left(V_{2}\right)_{1} \subset[4,+\infty)$. If not, we choose a larger value for $s_{2}$ and repeat the step. We are only interested in the value of the fourth component of the solution: since at all points of our interest $v_{4}^{\prime}<0$ (because $\left.v_{4}^{\prime}=v_{1} v_{4}\right)$, it suffices to compute the hull of the interval value of $v_{4}$ before and after the crossing of the hyperplane. We now have a rigorous proof that the intersection takes place at some $v_{4} \in\left[\min \left(V_{2}\right)_{4}, \max \left(V_{1}\right)_{4}\right]$ and this last interval (with the left bound rounded down and the right bound rounded up) is the value we display in Table (10). For the $\lambda^{*}$ computation we only display $\max \left(V_{1}\right)_{4}$ rounded down, since the other side of the interval does not have any meaning.

In order to check step 3 in Lemmas 13 and 14, it is not enough to check that the evolution of all points is in $A$ as defined in Subsection 6.2. Indeed, if the time step is large, it may happen that some trajectory leaves $A$ and reenters it in a single integration step. We have therefore to check at every time step that the whole rough enclosure $D$ as defined above is in $A$ and that the part of the set $A$ which is contained in the flow tube has a trivial topology, i.e. it does not have holes.

The round-off errors are taken care directly by Mathematica. Such errors may vary by changing computer and/or operating system, but since they are usually very small when compared to the wrapping effect, we expect that the proofs can be easily reproduced on any recent computer obtaining very similar bounds.

To perform the proofs we implemented a version of the whole algorithms in a combination of Mathematica and $\mathrm{C}++$ under the Linux O.S. More precisely, Mathematica has been used to handle all the data and to perform a few algorithms which are less demanding for the CPU, but more complicated to implement. Furthermore Mathematica has been used to make all numerical experiments and to draw the pictures. On the other hand $\mathrm{C}++$ has been used for the heavy interval arithmetic computations, where it offered a much higher speed. The connection between the two languages is obtained by MathLink. The verification of the whole proof takes a few days of CPU time on a machine equipped with an Athlon XP1700 processor. A full $\mathrm{C}++$ algorithm would reduce the time at the price of a much more complicated and less user-friendly programming. We think that this sharing of tasks is almost optimal, as far as computational speed and simplicity of programming and data handling are concerned. The reader who desires to reproduce the computer assisted proofs in this paper without writing the program can use the Mathematica notebook which is provided on the web at the address http://www1.mate.polimi.it/ gianni/aggm.nb. The notebook is provided with comments and instructions.

\section{References}

[ADN] S. Agmon, A. Douglis, L. Nirenberg, Estimates near the boundary for solutions of elliptic partial differential equations satisfying general boundary conditions. I, 
Commun. Pure Appl. Math. 12, 623-727 (1959)

[A1] H. Amann, Fixed point equations and nonlinear eigenvalue problems in ordered Banach spaces, SIAM Rev. 18, 620-709 (1976)

[A2] H. Amann, Ordinary differential equations. An introduction to nonlinear analysis, de Gruyter, Berlin etc. (1990)

[AZ] G. Arioli, P. Zgliczyński, Symbolic dynamics for the Hénon-Heiles Hamiltonian on the critical level, J. Diff. Equations 171, 173-202 (2001)

[BE] J. Bebernes, D. Eberly, Mathematical problems from combustion theory, Applied Mathematical Sciences 83, Springer, New York etc. (1989)

[B] T. Boggio, Sulle funzioni di Green d'ordine m, Rend. Circ. Mat. Palermo 20, 97-135 (1905)

[BCMR] H. Brezis, T. Cazenave, Y. Martel, A. Ramiandrisoa, Blow up for $u_{t}-\Delta u=g(u)$ revisited, Adv. Diff. Eq. 1, 73-90 (1996)

[BV] H. Brezis, J.L. Vazquez, Blow-up solutions of some nonlinear elliptic problems, Rev. Mat. Univ. Compl. Madrid 10, 443-468 (1997)

[C] S. Chandrasekhar, An introduction to the study of stellar structure, Dover Publ., New York (1967)

[CR] M.C. Crandall, P.H. Rabinowitz, Some continuation and variational methods for positive solutions of nonlinear elliptic eigenvalue problems, Arch. Rat. Mech. Anal. 58, 207-218 (1975)

[E] U. Elias, Nonoscillation and eventual disconjugacy, Proc. Amer. Math. Soc. 66, 269-275 (1977)

[GMP] T. Gallouet, F. Mignot, J.P. Puel, Quelques résultats sur le problème $-\Delta u=\lambda e^{u}$, C. R. Acad. Sci. Paris Sér. I 307, 289-292 (1988)

[GPP] J. García Azorero, I. Peral Alonso, J.P. Puel, Quasilinear problems with exponential growth in the reaction term, Nonlinear Anal. 22, 481-498 (1994)

[GG] F. Gazzola, H.-Ch. Grunau, Critical dimensions and higher order Sobolev inequalities with remainder terms, NoDEA Nonlinear Differential Equations Appl. 8, 35-44 (2001)

[GGM] F. Gazzola, H.-Ch. Grunau, E. Mitidieri, Hardy inequalities with optimal constants and remainder terms, to appear in Trans. Amer. Math. Soc.

[G] I.M. Gel'fand, Some problems in the theory of quasilinear equations, Section 15, due to G.I. Barenblatt, Amer. Math. Soc. Transl. II. Ser. 29, 295-381 (1963). Russian original: Uspekhi Mat. Nauk 14, 87-158 (1959)

[GNN] B. Gidas, W.M. Ni, L. Nirenberg, Symmetry and related properties via the maximum principle, Comm. Math. Phys. 68, 209-243 (1979) 
[GS] H.-Ch. Grunau, G. Sweers, Positivity properties of elliptic boundary value problems of higher order, Proc. 2nd World Congress of Nonlinear Analysts, Nonlinear Anal. T.M.A. 30, 5251-5258 (1997)

[HNW] E. Hairer, S.P. Nørsett, G. Wanner, Solving ordinary differential equations I - Nonstiff problems, 2nd printing of the 2nd edition, Springer, New York etc. (2000)

[J] J. Jacobsen, A Liouville-Gelfand equation for $k$-Hessian operators, Report AM 237, Pennsylvania State University (2001), available at:

http://www.math.psu.edu/ccma/reports.html

[JS] J. Jacobsen, K. Schmitt, The Liouville-Bratu-Gelfand problem for radial operators, J. Diff. Equations 184, 283-298 (2002)

[JL] D. Joseph, T.S. Lundgren, Quasilinear Dirichlet problems driven by positive sources, Arch. Rat. Mech. Anal. 49, 241-269 (1973)

[Li] P.L. Lions, On the existence of positive solutions of semilinear elliptic equations, SIAM Rev. 24, 441-467 (1982)

[Lo] R.J. Lohner, Computation of guaranteed enclosures for the solutions of ordinary initial and boundary value problems, in: Computational Ordinary Differential Equations, J.R. Cash, I. Gladwell (Eds.), pp. 425-435, Clarendon Press, Oxford, 1992

[Ma] Y. Martel, Uniquenes of weak extremal solutions for nonlinear elliptic problems, Houston J. Math. 23, 161-168 (1997)

[MP1] F. Mignot, J.P. Puel, Sur une classe de problèmes non linéaires avec nonlinéarité positive, croissante, convexe, Comm. Part. Diff. Eq. 5, 791-836 (1980)

[MP2] F. Mignot, J.P. Puel, Solution radiale singulière de $-\Delta u=\lambda e^{u}$, C. R. Acad. Sci. Paris Sér. I 307, 379-382 (1988)

[Mo] J.J. Moreau, Décomposition orthogonale d'un espace hilbertien selon deux cônes mutuellement polaires, C. R. Acad. Sci. Paris 255, 238-240 (1962)

[MZ] M. Mrozek, P. Zgliczyński, Set arithmetic and the enclosing problem in dynamics, Annales Polonici Mathematici, in press

[PT] L.A. Peletier, W.C. Troy, Spatial patterns. Higher order models in physics and mechanics, Progress in Nonlinear Differential Equations and their Applications 45, Birkhäuser, Boston etc. (2001)

[P] S.I. Pohožaev, Eigenfunctions of the equation $\Delta u+\lambda f(u)=0$, Soviet Math. Doklady 6, 1408-1411 (1965)

[PS] P. Pucci, J. Serrin, A general variational identity, Indiana Univ. Math. J. 35, 681$703(1986)$

[So] R. Soranzo, A priori estimates and existence of positive solutions of a superlinear polyharmonic equation, Dyn. Syst. Appl. 3, 465-487 (1994) 
[Sw] G. Sweers, No Gidas-Ni-Nirenberg type result for biharmonic problems, Math. Nachr., in press

[We] J. Wei, Asymptotic behavior of a nonlinear fourth order eigenvalue problem, Comm. Part. Diff. Eq. 21, 1451-1467 (1996)

[Wi] Ch. Wieners, Numerische Existenzbeweise für schwache Lösungen nichtlinearer elliptischer Randwertaufgaben (Numerical proofs of existence of weak solutions of nonlinear elliptic boundary value problems), PhD thesis, Univ. Cologne (1994)

Gianni Arioli

Dipartimento di Matematica del Politecnico

via Bonardi 9, 20133 Milano (Italy)

e-mail: gianni@mate.polimi.it

Filippo Gazzola

Dipartimento di Matematica del Politecnico

via Bonardi 9, 20133 Milano (Italy)

e-mail: gazzola@mate.polimi.it

Hans-Christoph Grunau

Fakultät für Mathematik, Otto-von-Guericke-Universität

Postfach 4120, 39016 Magdeburg (Germany)

e-mail: Hans-Christoph.Grunau@mathematik.uni-magdeburg.de

Enzo Mitidieri

Dipartimento di Scienze Matematiche

via A. Valerio 12/1, 34100 Trieste (Italy)

e-mail: mitidier@univ.trieste.it 